\title{
Statistical image reconstruction from limited projection data with intensity priors
}

\author{
Essam A Rashed ${ }^{1,2}$ and Hiroyuki Kudo ${ }^{1}$ \\ ${ }^{1}$ Department of Computer Science, Graduate School of Systems and Information \\ Engineering, University of Tsukuba, Tennoudai 1-1-1, Tsukuba 305-8573, Japan \\ ${ }^{2}$ Department of Mathematics, Faculty of Science, Suez Canal University, Ismailia \\ 41522, Egypt \\ E-mail: essam@imagelab.cs.tsukuba.ac.jp
}

\begin{abstract}
The radiation dose generated from x-ray computed tomography (CT) scans and its responsibility for increasing the risk of malignancy became a major concern in the medical imaging community. Accordingly, investigating possible approaches for image reconstruction from low-dose imaging protocols, that minimize the patient radiation exposure without affecting image quality, has become an issue of interest. Statistical reconstruction (SR) methods are known to achieve a superior image quality compared with conventional analytical methods. Effective physical noise modeling and possibilities to incorporate priors in the image reconstruction problem are the main advantages of the SR methods. Nevertheless, the high computation cost limits its wide use in clinical scanners. This article presents a framework for $\mathrm{SR}$ in x-ray CT when the angular sampling rate of the projection data is low. The proposed framework is based on the fact that, in many CT imaging applications, some physical and anatomical structures and the corresponding attenuation information of the scanned object can be a priori known. Therefore, the x-ray attenuation distribution in some regions of the object can be expected prior to the reconstruction. Under this assumption, the proposed method is developed by incorporate this prior information into the image reconstruction objective function to suppress streak artifacts. We limit the prior information to only a set of intensity values that represent the average intensity of the normal and expected homogeneous regions within the scanned object. This prior information can be easily computed in several x-ray CT applications. Considering the theory of compressed sensing, the objective function is formulated using $\ell_{1}$ norm distance between the reconstructed image and the available intensity priors. Experimental comparative studies applied to simulated data and real data, are used to evaluate the proposed method. The comparison indicates a significant improvement in image quality when the proposed method is used.
\end{abstract}

Submitted to: Phys. Med. Biol. 


\section{Introduction}

X-ray computed tomography (CT) imaging is generally used through the reconstruction of an object function from its projection data. The projection data, measured by a detector array, represents the internal structure through the attenuation map of the xray photons. The sampling rate and data-acquisition orbit have a strong influence on the exactness and stability of the image reconstruction process. It is well known that undersampling of projection views converts the image reconstruction inverse problem into ill-posed one (Natterer 1986, Herman and Kuba 1999), where the reconstructed image sufferers from different types of image artifacts that vary according to the availability of projection data (Joseph and Schulz 1980 Rangayyan et al 1985). A common data limitation problem in clinical and industrial applications is the limited view problem. The term limited view, which is also called sparse projections, refers to the case where the measured projection data is obtained over a small number of equally/unequally distant angles less than what is traditionally required by the Nyquist sampling rule (Natterer 1986). In medical imaging, as well as several industrial applications, it is required to reconstruct an artifact-free image from a limited view projection data. When the target object is presented in binary form (e.g. 0/1), exact reconstruction can be achieved from small number of projections (Herman and Kuba 2007). Unfortunately, this assumption is not valid in several real CT applications. The problem of image reconstruction from small projections had been studied, and many reconstruction algorithms are already proposed in the literature, see e.g. (Huesman 1977, Brooks et al 1979, Nassi et al 1982, Payot et al 1997, Galigekere et al 1999, Li et al 2004, Sidky et al 2006, Herman and Davidi 2008, Hansis et al 2008).

The wide spread of the x-ray CT scanners worldwide and their extensive usage in medical diagnosis for many diseases has led to a significant dose increase to the community. The minimization of radiation exposure in CT imaging had become a key requirement in many imaging applications, especially for pediatric (Brenner and Hall 2007, Yu et al 2009). For example, in image-guided radiation therapy (IGRT), patients are usually subject to multiple scans that accumulate the dose to a risky level. However, decreasing x-ray beam intensity usually increases the statistical noise in the reconstructed images, which reduces the ability to extract useful information from these images. One potential approach, that achieves the objective of ionizing radiation reduction, while providing patients with more comfortable short time imaging, is to under-sample the number of projection views.

Statistical reconstruction (SR) has been used for long time in emission tomography for its ability to reduce statistical noise in reconstructed images. The correct noise modeling and the ability to incorporate a priori information in the image reconstruction objective function are the key reasons for being used in several in-duty PET and SPECT imaging equipments. However, in x-ray CT, conventional methods derived from analytical inversion formulae, such as the standard filtered backprojection (FBP), are still the methods of choice in clinical scanners. The main reason is the expensive 
computation cost of SR methods when it is applied to relatively large photon counts used in x-ray CT. Recently, the use of SR in transmission CT has taken much interest due to the rapid growth of the computing power and the use of high-speed GPU processing units in image reconstruction.

In CT imaging, we measure the attenuation of x-ray within the scanned object. In this imaging modality, it is known that the variation of the attenuation coefficients, it is also called intensity, within the same homogenous object is rather small. The incorporation of a priori known information, to improve images reconstructed from limited projection data in CT, have been extensively studied in the past two decades, see e.g. (Sauer et al 1994, Kolehmainen et al 2003, Siltanen et al 2003, Chen et al 2008, Leng et al 2008, Rashed and Kudo 2011). The nature of the prior information required for exact reconstruction is not clear yet. Thus, it is interesting to quantitatively investigate how different types of prior information contribute to the image quality in various data limitation problems. We can classify the possible prior information into the following three categories: (1) full reference image (intensity value + pixel position), (2) boundaries and support information (pixel position only), and (3) attenuation information (intensity value only). The first category considers the case where a priori information is obtained from a reference image generated prior to the reconstruction. The term reference image usually refers to an image that includes basic and normal structures of the scanned object. Reference image may be obtained through an earlier scan of the patient, segmentation of neighboring slices, or blurred image reconstructed from all measured projection data in gated-CT. The second category describes the use of a priori information corresponding to the boundaries of uniform regions without further spatial information. This includes the well-known quadratic smoothing penalty. The third category corresponds to the case where a priori information is limited to a set of intensity values representing uniform regions of the object without additional knowledge about the pixel positions. In this work, we study the use of prior information corresponding to the third category.

In this paper, we propose a Bayesian framework for SR from limited view projection data. We assume that a small number of intensity values that represent average value of expected homogenous regions (organs) are a priori known or estimated with high accuracy. For convenience, we refer to this prior information as intensity prior throughout the paper. We design the objective function such that it includes a distance function based on $\ell_{1}$ norm to the intensity prior. The resulting SR method is evaluated using simulated data and real data with different settings for the prior.

This paper is organized as follows. In section 2, we briefly introduce the imaging model for SR in transmission CT. The proposed approach is detailed, and the image reconstruction algorithm is developed in section 3. The experimental data is presented and analyzed in section 4 and followed by discussion and conclusion in section 5 . 


\section{Imaging model for statistical reconstruction}

\subsection{Statistical image reconstruction}

In transmission CT, the x-ray beam is radiated to the target object where a portion of the radiation is absorbed by the object while the remaining portion transmits through it. The transmitted portion of the radiated photons is measured by an array of detectors located on the opposite side of the x-ray tube. The attenuation map $\mu$ of the object can be identified according to the Beer's law of attenuation (Lange and Carson 1984):

$$
I=I_{0} \exp \left(-\int_{s} \mu d s\right)
$$

where $I$ and $I_{0}$ are the photon counts, and the blank scan measured at a single detector bin, respectively, with $s$ represents the virtual ray path from the tube focal point to the detector bin. Assuming that the attenuation value is constant within small size image pixels, the transmission x-ray CT can be described in a discrete form using the following statistical model:

$$
y_{i} \approx \operatorname{Poisson}\left(b_{i} \exp \left(-\sum_{j=1}^{n} a_{i j} \mu_{j}\right)\right)
$$

where $\mu=\left(\mu_{1}, \ldots, \mu_{n}\right)$ is the image vector representing the attenuation coefficients of the imaged object, $y=\left(y_{1}, \ldots, y_{m}\right)$ is a vector represent the raw detector measurements with the corresponding blank scan $b=\left(b_{1}, \ldots, b_{m}\right)$ and $A=\left\{a_{i j}\right\}$ is the $m \times n$ system matrix that models the imaging system. In the statistical model in equation (2), we consider the use of a monochromatic x-ray spectrum and ignore the effect of scattered photons, for simplicity. The log-likelihood function for the observed photon counts, as described in literature, is given by:

$l(\mu)=-L(\mu)=\sum_{i=1}^{m}\left[y_{i} \log \left(b_{i}\right)-y_{i} \sum_{j=1}^{n} a_{i j} \mu_{j}-\log \left(y_{i} !\right)-b_{i} \exp \left(-\sum_{j=1}^{n} a_{i j} \mu_{j}\right)\right]$.

The solution of the image reconstruction problem is found through a maximization

of the log-likelihood function after ignoring the irrelevant terms. The maximum likelihood solution of the statistical reconstruction problem is found by:

$$
\mu^{*}=\arg \min _{\mu \geq 0} L(\mu), \quad L(\mu)=\sum_{i=1}^{m}\left[b_{i} \exp \left(-\left\langle a_{i}, \mu\right\rangle\right)+y_{i}\left\langle a_{i}, \mu\right\rangle\right],
$$

where $\left\langle a_{i}, \mu\right\rangle=\sum_{j=1}^{n} a_{i j} \mu_{j}$ is the inner product of the $i$ th row of matrix $A$ and image vector $\mu$. When the acquired data $y$ are limited such that $m \ll n$, the image reconstruction becomes an ill-posed problem and achieving a reliable and feasible solution is challenging. To overcome this problem, the well-known Bayesian methods, that include a priori information on the scanned object into account, are used. The solution of the image reconstruction problem is found through maximizing a posteriori (MAP) function:

$$
P(\mu \mid y)=\frac{P(y \mid \mu) P(\mu)}{P(y)},
$$


and the maximum a priori solution of the reconstruction problem is found by:

$$
\mu^{*}=\arg \min _{\mu \geq 0} f_{\beta}(\mu), \quad f_{\beta}(\mu)=L(\mu)+\beta U(\mu),
$$

where $U(\mu)$ is known as the penalty term or the regularization function that presents prior knowledge of the image object and $\beta$ is a hyper-parameter that controls the power of the regulation term.

\subsection{Compressed Sensing}

Since the introduction of the compressed sensing (CS) principle (Candès and Tao 2006, Donoho 2006), several approaches were presented to solve the inverse problem for image reconstruction from a small number of projection views. The main approach is based on the minimization of the objective function that includes $\ell_{1} / \ell_{0}$ norm of a sparse representation of the target object. This formulation of the objective function can effectively find the sparse solution from feasible ones. When the object is presented in a sparse form, the number of projections required for a good estimation is significantly less than that of the traditional Nyquest sampling criteria. This approach was presented early and was used to develop an image reconstruction algorithm for blood vessel imaging from a small number of projection views ( $\mathrm{Li}$ et al 2002). When the target object is originally sparse, the image reconstruction problem is formulated generally as the minimization of $N(\mu)$ subject to data fidelity terms. Where $N($.$) is a norm function$ such as $\ell_{1} / \ell_{0}$ norms. However, in many cases of x-ray CT applications, the target object is non-sparse. The image reconstruction problem is then formulated to minimize $N(\Phi(\mu))$, where $\Phi($.$) is a sparsification transform such as general gradient, wavelets, or$ total-variation (TV) norm. The TV norm was introduced for the first time by Rudin et al (1992) as an effective tool for image denoise. The TV norm is known for its edge preservation property providing that it is based on non-quadratic function and this is the main reason that TV-based image reconstruction algorithms have gained much attention recently. For example, it is used for SPECT imaging (Persson et al 2001), Cone-beam CT (Sidky and Pan 2008) and cardiac gated micro-CT (Song et al 2007). Interesting comparison studies between the TV-based approaches and SR methods were recently presented by Tang et al (2009) and Bian et al (2010).

However, the use of straight TV models for realistic image reconstruction has been shown to produce artificial patches that reduce the image quality and their possibility to be used in clinical equipment as well (Fahimian et al 2010). Furthermore, it is known that TV-based methods may result in losing small size and low-contrast structures during the reconstruction. Moreover, the TV-minimization is usually implemented through iteration procedures, which considerably increase the computation cost of the image reconstruction process, especially in $3 \mathrm{D} / 4 \mathrm{D}$ reconstruction. In general, when the projection data are highly under-sampled, the image reconstruction becomes underdetermined problem and the achievement of exact reconstruction is not theoretically possible (Herman and Davidi 2008). Additional terms, added to the 


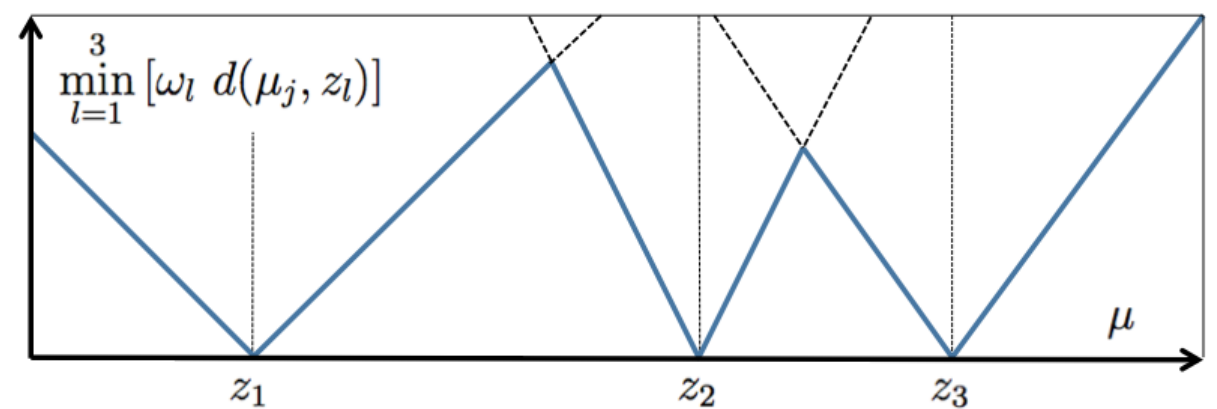

Figure 1. The proposed penalty function in equation (8) corresponding to $L=3$.

objective function, can lead to a more accurate reconstruction. Here, we introduce a simple and easy-to-compute prior information which we call intensity prior. As it will be shown later through the experimental studies, intensity prior can be easily estimated, and it contributes effectively to the image quality without affecting the contrast.

\subsection{MAP reconstruction and intensity prior}

The penalty term $U(\mu)$ in equation (6) can be formulated using several approaches. It is common in nuclear imaging to introduce a penalty term in the image reconstruction objective function for the benefit of obtaining a data consistent image with less noise. However, it is difficult to introduce a prior knowledge that fits with the imaging applications. For example, a commonly used quadratic smoothing penalty is defined as:

$$
U(\mu)=\sum_{j, j^{\prime} \in C} \omega_{j, j^{\prime}}\left(\mu_{j}-\mu_{j^{\prime}}\right)^{2}
$$

where $C$ is a set of neighboring pixels and $\omega_{j, j^{\prime}}$ is a weight assigned to each pair of pixels $\left(j, j^{\prime}\right)$. Using the quadratic smoothing penalty can effectively suppress noise but on the expense of preserving sharp edges.

In this paper, we introduce the penalty term as a distance function between the reconstructed image $\mu$ and the available intensity values. In x-ray $\mathrm{CT}$, the attenuation coefficients within the same region (organ) are almost uniform and can be a priori known within a specific range that slightly varies based on the imaging application. For example, it is not difficult to estimate, with high accuracy, the attenuation coefficients of normal organs appearing in a chest CT imaging of a mature patient. The distance function can take various forms based on image properties and the nature of available a priori information. Considering the intensity prior, the proposed distance function is formulated as:

$$
U(\mu) \equiv D(\mu, z)=\sum_{j=1}^{n} \min _{l=1}^{L}\left[\omega_{l} d\left(\mu_{j}, z_{l}\right)\right],
$$

where $z=\left(z_{1}, \ldots, z_{L}\right)$ are the known intensity values arranged in ascending order (i.e. $\left.z_{1}<z_{2}<\ldots<z_{L}\right), \omega_{1}, \ldots, \omega_{L}$ is a set of empirically determined parameters 
that quantitatively represent the corresponding intensity values, and $d(.,$.$) is a single-$ variable distance function. This distance function should be carefully selected because it has a strong influence on the quality of the reconstructed image. A possible useful choice is the $\ell_{1}$ norm distance such that:

$$
d(a, b)=|a-b|
$$

The most advantage feature, of the proposed penalty function in equation (8), is that the required a priori information is limited to only a small number of intensity values, which can be easily available in several clinical applications.

\section{Methods}

\subsection{Reconstruction algorithm}

The main difficulty in minimizing the objective function in equation (6) is that the proposed penalty term in equation (8) is based on $\ell_{1}$ norm, which is neither convex nor differentiable. Therefore, it is not possible to use ordinary gradient-type iterative method to optimize the objective function. Alternatively, the well-known majorizarionminimization (MM) strategy (Fessler and Hero 1995, Daubechies et al 2004) is used to derive separable quadratic function. At each iteration $k$, the non-separable part of the main objective function in equation (6) is approximated by a separable quadratic function $\tilde{L}\left(\mu ; \mu^{k}\right)$ around $\mu=\mu^{k}$ such that:

$$
\begin{aligned}
& \tilde{L}\left(\mu^{k} ; \mu^{k}\right)=L\left(\mu^{k}\right), \\
& \partial \tilde{L}\left(\mu ; \mu^{k}\right) /\left.\partial \mu_{j}\right|_{\mu=\mu^{k}}=\partial L(\mu) /\left.\partial \mu_{j}\right|_{\mu=\mu^{k}} .
\end{aligned}
$$

The approximate quadratic majorization to $L(\mu)$ is formulated as:

$$
\begin{aligned}
\tilde{L}\left(\mu ; \mu^{k}\right) & =L\left(\mu^{k}\right)+\left.\nabla L(\mu)\right|_{\mu=\mu^{k}}\left(\mu-\mu^{k}\right)^{\top}+\frac{1}{2} Q\left(\mu^{k}\right)\left(\mu_{j}-\mu_{j}^{k}\right)^{2} \\
& =\frac{1}{2} \sum_{j=1}^{n} \frac{\sum_{i=1}^{m} a_{i j}\left\langle a_{i}, \mu^{k}\right\rangle b_{i} \exp \left(-\left\langle a_{i}, \mu^{k}\right\rangle\right)}{\mu_{j}^{k}}\left(\mu_{j}-p_{j}\right)^{2}+T\left(\mu^{k}\right) .
\end{aligned}
$$

where $T\left(\mu^{k}\right)$ is the term independent of $\mu$,

$$
Q\left(\mu^{k}\right)=\sum_{j=1}^{n} \frac{\sum_{i=1}^{m} a_{i j}\left\langle a_{i}, \mu^{k}\right\rangle b_{i} \exp \left(-\left\langle a_{i}, \mu^{k}\right\rangle\right)}{\mu_{j}^{k}},
$$

and

$$
p_{j}=\mu_{j}^{k}+\mu_{j}^{k} \frac{\sum_{i=1}^{m} a_{i j}\left(b_{i} \exp \left(-\left\langle a_{i}, \mu^{k}\right\rangle\right)-y_{i}\right)}{\sum_{i=1}^{m} a_{i j}\left\langle a_{i}, \mu^{k}\right\rangle b_{i} \exp \left(-\left\langle a_{i}, \mu^{k}\right\rangle\right)} .
$$

The resulting separable objective function $\tilde{f}_{\beta}\left(\mu ; \mu^{k}\right)=\tilde{L}\left(\mu ; \mu^{k}\right)+\beta U(\mu)$ is minimized analytically to obtain the next iterate $\mu^{k+1}$. This approximation is useful to obtain a separable objective function when the penalty term is non-differentiable such as the case of $\ell_{1}$ norm distance. We consider the proposed penalty term of intensity 
information in equation (8), in which the separable objective function $\tilde{f}_{\beta}\left(\mu ; \mu^{k}\right)$ can be written as:

$$
\begin{aligned}
& \tilde{f}_{\beta}\left(\mu ; \mu^{k}\right)=\sum_{j=1}^{n} \beta \omega_{h}\left[c_{j, h}\left(\mu_{j}-p_{j}\right)^{2}+d\left(\mu_{j}, z_{h}\right)\right]+T\left(\mu^{k}\right), \\
& h=\left\{h \in\{1, \ldots, L\}: d\left(\mu_{j}, z_{h}\right)=\min _{l=1}^{L} d\left(\mu_{j}, z_{l}\right)\right\} \\
& c_{j, h}=\frac{1}{2 \beta \omega_{h} \mu_{j}^{k}} \sum_{i=1}^{m} a_{i j}\left\langle a_{i}, \mu^{k}\right\rangle b_{i} \exp \left(-\left\langle a_{i}, \mu^{k}\right\rangle\right) .
\end{aligned}
$$

The proposed algorithm, which we called intensity-based MAP ( $i \mathrm{MAP})$, for transmission tomography is implemented through the following steps:

(i) (Initialization step) Estimate the intensity prior $z=\left(z_{1}, \ldots, z_{L}\right)$, set the initial image $\mu^{0}$ to a uniform positive value and set the iteration number $k \rightarrow 0$.

(ii) (Majorization step) The objective function $f(\mu)$ is approximately majorized around the current estimate $\mu^{k}$ using equation (13) to the separable function in equation (16).

(iii) (Minimization) The separable objective function $\tilde{f}\left(\mu ; \mu^{k}\right)$ is minimized over $\mu \geq 0$ to obtain the image estimate $\mu^{k+1}$ in the next iterate:

$$
q=\arg \min _{\mu \geq 0} \tilde{f}_{\beta}\left(\mu ; \mu^{k}\right), \quad \mu_{j}^{k+1}=\max \left(q_{j}, \epsilon\right)
$$

where $\epsilon$ is a small value to guarantee that $\mu_{j}^{k+1}>0$.

(iv) (Iterate condition) Set iteration number $k \rightarrow k+1$, repeat steps (i)-(iii) until reaching to a stopping criterion.

The main theoretical framework for the derivation of the $i \mathrm{MAP}$ algorithm is the same as that presented in the earlier work for anatomical-MAP-EM method for PET/SPECT imaging (Mameuda and Kudo 2007). However, the present method investigates a different imaging problem in transmission $\mathrm{CT}$, where the essential target of the previous work was to reconstruct PET/SPECT images with better noise/contrast properties. Moreover, the intensity prior, which is more appropriate for the transmission CT imaging, is an original contribution of this paper.

It is known that, in generic $\ell_{1}$ norm prior, the convergence of the iterative algorithm is guaranteed (Daubechies et al 2004, Loris 2009). In this paper, we have used the MM strategy to generate approximate separable function for which the convergence properties of the proposed algorithm are not clear. However, the same framework can be used with exact surrogate function presented by Erdǒgan and Fessler (1999). In this case, the algorithm monotonically decreases the objective function towards a local minimum. Nevertheless, as the objective function is non-convex, there is no proof that the algorithm will achieve the global convergence. Recently, it becomes interesting to study the performance of similar algorithms and some papers have discussed the convergence properties of iterative hard thresholding (e.g. Blumensath and Davies 2010). 


\subsection{Intensity multi-thresholding}

The minimization in equation (18) can now be implemented directly. It is easy to prove that the optimization of the following simple separable objective function:

$$
x^{*}=\arg \min _{x} \frac{\alpha}{2}(x-a)^{2}+\beta|x-b|,
$$

can be found through the following simple soft-thresholding function:

$$
x^{*}= \begin{cases}a+\beta / \alpha & (a<b-\beta / \alpha) \\ b & (b-\beta / \alpha \leq a \leq b+\beta / \alpha) . \\ a-\beta / \alpha & (a>b+\beta / \alpha)\end{cases}
$$

In the same way, it is direct to estimate a multi-thresholding function corresponding to the minimization of the objective function in equation (16). Therefore, the iterative reconstruction algorithm is implemented through the following simple procedure. First, we compute the term $p_{j}$ in equation (15) which is same as a single iteration of the Convex algorithm (Lange and Fessler 1995). Then, the minimization is implemented through the following multi-thresholding function:

$q_{j}= \begin{cases}p_{j}+1 /\left(2 c_{j, l}\right) & \left(s_{l-1}<p_{j}<z_{j, l}^{-}\right) \\ z_{l} & \left(\max \left(z_{j, l}^{-}, s_{l-1}\right) \leq p_{j} \leq \min \left(z_{j, l}^{+}, s_{l}\right)\right) \quad,(l=1, \ldots, L) . \\ p_{j}-1 /\left(2 c_{j, l}\right) & \left(z_{j, l}^{+}<p_{j} \leq s_{l}\right)\end{cases}$

with $z_{j, l}^{+}=z_{l}+1 /\left(2 c_{j, l}\right), z_{j, l}^{-}=z_{l}-1 /\left(2 c_{j, l}\right), s_{l}=\left(\omega_{l} z_{l}+\omega_{l+1} z_{l+1}\right) /\left(\omega_{l}+\omega_{l+1}\right), s_{0}=-\infty$ and $s_{L}=\infty$.

This multi-thresholding function, can be expressed as a combination of multiple successive soft-thresholding functions, each is implemented around a single value of the a priori known intensity values $z_{l}, l=1, \ldots, L$. The practical interpretation of this thresholding operation is as follows. If the computed pixel value $p_{j}$ is closed to the intensity value $z_{l}$ (i.e. located inside the thresholding window controlled by the parameter $\omega_{l}$ ), the pixel value is trimmed to $z_{l}$. Otherwise, the pixel value $p_{j}$ is shifted softly towards the closest value of $z_{l}$. Illustration of the multi-thresholding functions is in figure 2 .

In MAP reconstruction algorithms, the hyper-parameter $\beta$ in equation (6) is known as the regularization parameter which handles the strength of the prior term. In the $i \mathrm{MAP}$ algorithm, $\beta$ controls the size of the thresholding window in the multithresholding function in (21). Obviously, the value of $\beta$ should be carefully selected. On the one hand, if $\beta$ is assigned to a rather large value, some low-frequency details in the reconstructed image will be lost due to the strong thresholding. On the other hand, if $\beta$ is relatively small, the merit of thresholding is weak and the proposed method behaves similar to the conventional reconstruction algorithm. We propose to use a dynamic value for this parameter by starting with a relatively large value and then gradually decrease it according to the following rule.

$$
\lim _{k \rightarrow \infty} \beta_{k}=\delta \quad \text { and } \quad \sum_{k=0}^{\infty} \beta_{k}=\infty .
$$




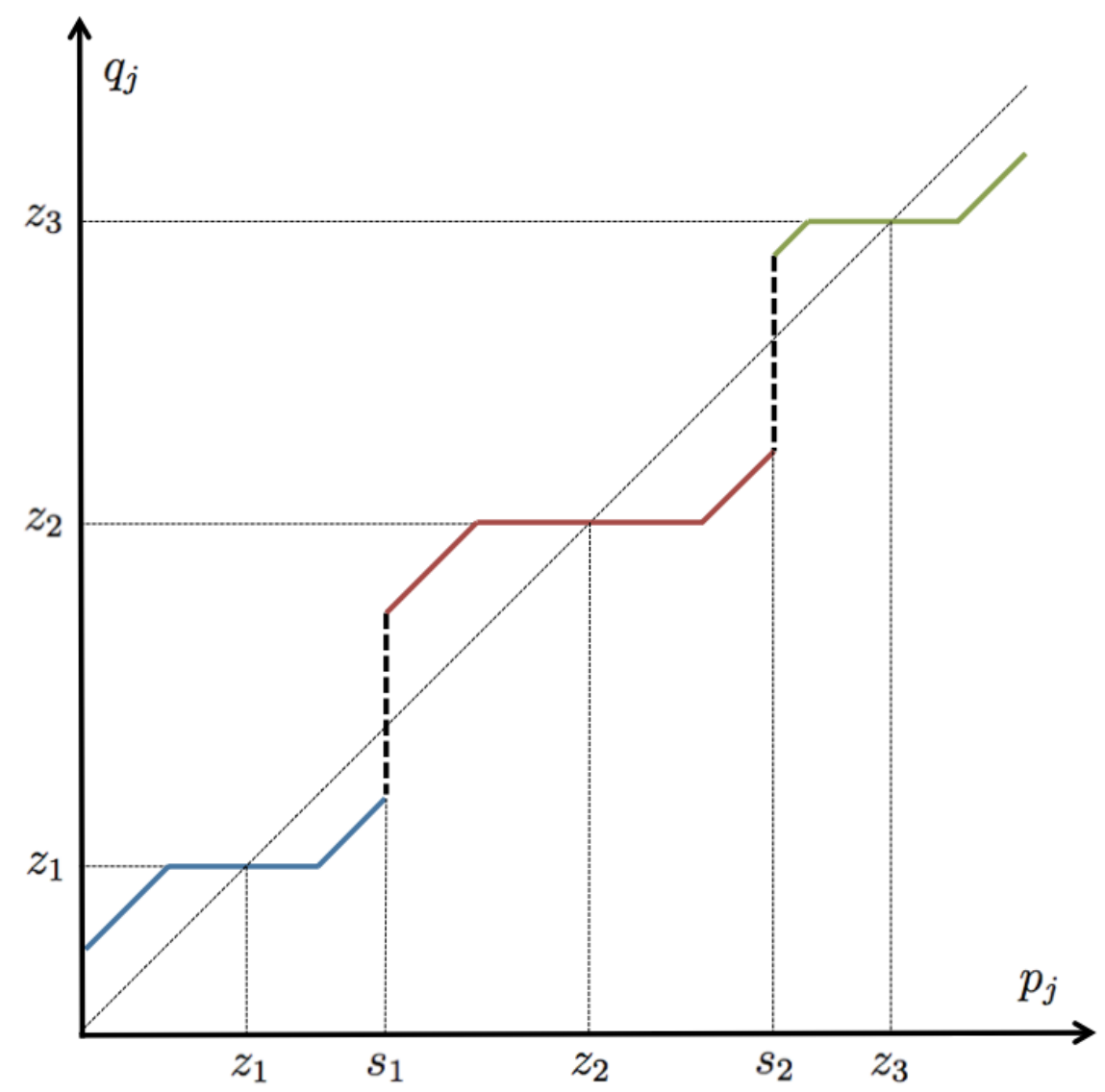

Figure 2. The multi-thresholding function in equation (21) with $L=3$.

where $\delta$ is a small value empirically defined to represent the noise magnitude, and equals to zero for noise-free data. In the experimental studies presented in this paper, we have used ordered subsets version of the proposed algorithm (OS-iMAP) to speed up the reconstruction, and the under-relaxation parameter is implemented using the following simple rule:

$$
\beta_{k}=\left(k_{\max }+1\right) \beta /(k+1) .
$$

The use of this dynamic hyper-parameter is useful to speed up the convergence in the practical implementation. This approach is based on the observation that, in the early iterations, a strong thresholding is required to remove acute streak artifacts, and a weak thresholding is preferred later to retrieve missing small-size and low-contrast image details. Moreover, it is known that the use of under-relaxation parameter improves the convergence properties of ordered subsets reconstruction by removing the limit cycle behavior (Kole 2005).

The proposed $i$ MAP algorithm has various advantages. First, the penalty function for intensity prior in equation (8) can be easily embedded in several famous transmission SR algorithms, for example, the EM algorithm (Lange and Carson 1984), gradient algorithm (Lange et al 1987) and its ordered subset version (Beekman and Kamphuis 
Table 1. Standard attenuation values of body structures and other objects, measured in Hounsfield units (HU).

\begin{tabular}{ll}
\hline Anatomical structure & HU range \\
\hline air & -1000 \\
lung & $-900:-800$ \\
fatty-tissue & $-100:-50$ \\
water & 0 \\
blood & $30: 50$ \\
muscles & $30: 80$ \\
contrast enhanced blood & $200: 500$ \\
calcification & $130: 500$ \\
bone & $500: 1500$ \\
metal & $>1000$ \\
\hline
\end{tabular}

2001). Second, the computation cost is almost same as the original method since the cost of the multi-thresholding step can be ignored relative to the computation of a single iteration of the original reconstruction algorithm. Third, the required intensity prior, as we will present in next section, can be easily estimated with high accuracy in many imaging situations.

\subsection{Estimation of intensity prior}

In the $i \mathrm{MAP}$ algorithm presented above, we assume that a set of intensity values $z$ is a priori known. In this section, we discuss possible approaches to compute the intensity prior.

3.3.1. application-based estimation In many x-ray CT applications, the anatomical structure as well as the attenuation coefficient of the target object can be estimated with high accuracy. The knowledge of the tube current energy and the exposure time used in the imaging equipment can provide an accurate limited range for the expected intensity values corresponding to the anatomy of the scanned object. For example, the standard intensity values, in Hounsfield units (HU), expected from average radiation dose used in clinical CT scanners are shown in table 1 (Ohnesorge et al 2006). These values can be simply used as intensity prior in several CT imaging applications with weighting values $\omega$ that approximately represent the power of each intensity value.

3.3.2. image-based estimation In some CT applications, such as IGRT, the patient is subject to multiple scans during a short time of treatment, which significantly increase the patient dose to a risky level. A possible alternative imaging protocol, that minimizes the dose and also reduces the scan time can be as follows. First, the patient is imaged with a normal dose for a single time. The reconstructed image is used to estimate the intensity values that represent different regions of interest. The later periodic scans 


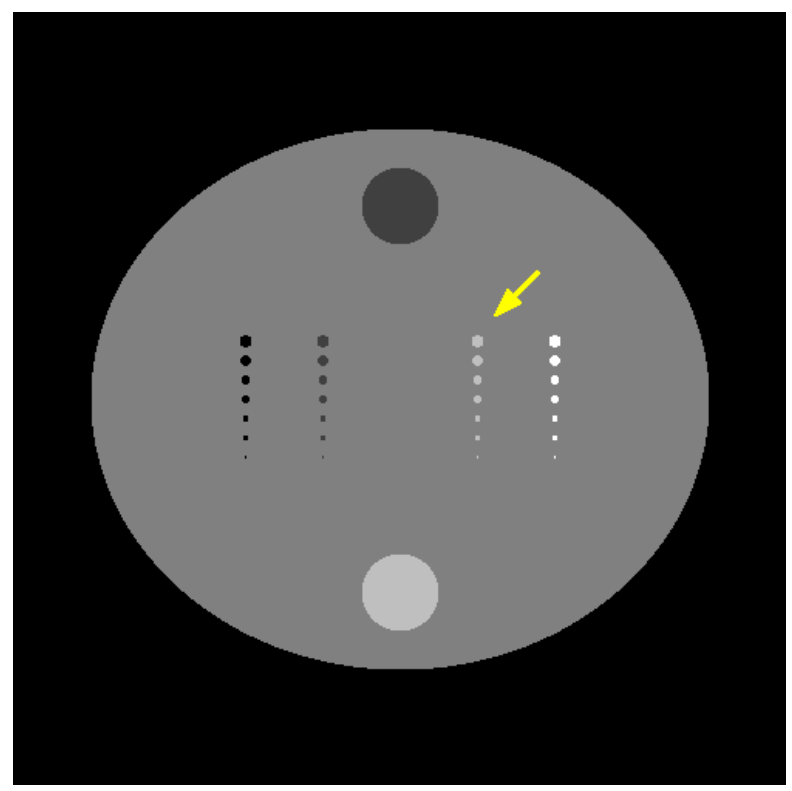

Figure 3. Digital phantom used in the simulation studies. The arrow is pointing to the column of seven resolution inserts used to compute the image contrast.

are then implemented through a low-dose or small views scan, which can be employed using intensity prior with the proposed method. Furthermore, it is possible to estimate the intensity values from a segmented image obtained from an earlier scan of the same patient or another patient. Moreover, in multi-slice CT imaging, the patient dose can be modulated by using hybrid scan, which alternates conventional scan in a single rotation and low-dose scan in later few ones. Intensity values obtained from slices reconstructed from normal dose data, can be used for the reconstruction of the neighbor slices using data acquired from low-dose scans.

3.3.3. data-based estimation If the a priori information about the internal anatomy of the scanned object is unknown, the intensity values can be estimated directly from the projection data through a clustering of image estimate. As shown later in the experimental studies, it is possible to estimate intensity values during the image reconstruction.

\section{Experimental results}

In this section, the proposed method is evaluated. The experiments are implemented using simulated phantom and real data obtained from different x-ray CT imaging applications.

\subsection{Assessment of image quality}

Throughout the experimental data presented here, the image quality is measured using several methods. Along with each reconstruction result, the corresponding true object in 
Table 2. Parameters of digital phantom shown in figure 3. Objects from number 4 to 10 are repeated with $\mathrm{a}=(-0.4,-0.2,0.2,0.4)$ and $\mathrm{b}=(0.0,0.5,1.5,2.0)$, respectively.

\begin{tabular}{lllll}
\hline Index & Center coordinates & Major axis & Minor axis & Density $\left(\mathrm{cm}^{-1}\right)$ \\
\hline 1 & $(0.0,0.0)$ & 0.8 & 0.7 & 1.0 \\
2 & $(0.0,0.5)$ & 0.1 & 0.1 & 0.5 \\
3 & $(0.0,-0.5)$ & 0.1 & 0.1 & 1.5 \\
4 & $(\mathrm{a},-0.15)$ & 0.016 & 0.016 & $\mathrm{~b}$ \\
5 & $(\mathrm{a},-0.10)$ & 0.014 & 0.014 & $\mathrm{~b}$ \\
6 & $(\mathrm{a},-0.05)$ & 0.012 & 0.012 & $\mathrm{~b}$ \\
7 & $(\mathrm{a}, 0.00)$ & 0.010 & 0.010 & $\mathrm{~b}$ \\
8 & $(\mathrm{a}, 0.05)$ & 0.008 & 0.008 & $\mathrm{~b}$ \\
9 & $(\mathrm{a}, 0.10)$ & 0.006 & 0.006 & $\mathrm{~b}$ \\
10 & $(\mathrm{a}, 0.15)$ & 0.004 & 0.004 & $\mathrm{~b}$ \\
\hline
\end{tabular}

simulated data or the FBP reconstruction from complete projection data, is illustrated for visual evaluation. We also calculate the root mean square error (RMSE) for image quality evaluation:

$$
\operatorname{RMSE}(\mu)=\sqrt{\sum_{j=1}^{n}\left(\mu_{j}-\overline{\mu_{j}}\right)^{2} / \sum_{j=1}^{n} \overline{\mu_{j}}}
$$

where $\bar{\mu}$ represents the true object or the FBP image reconstructed from complete data. We also measure the contrast of the reconstructed image using the following formula:

$$
\operatorname{contrast}(\mu)=\frac{\left|\mu_{s}-\mu_{b}\right|}{\mu_{s}+\mu_{b}}
$$

where $\mu_{s}$ and $\mu_{b}$ are the average intensity values of selected resolution insert pixels and background pixels, respectively.

\subsection{Simulation studies}

In the first simulation, we have used a digital phantom shown in figure 3 . The phantom size was $100 \mathrm{~mm}$ consisting of a uniform background with resolution inserts of different size and contrast. The size of the inserts is ranged from $0.4 \mathrm{~mm}$ to $1.6 \mathrm{~mm}$ arranged in four columns each of seven inserts. Two additional inserts of $10 \mathrm{~mm}$ are also added to the phantom. The parameters of this phantom are shown in table 2. The image grid was set to $500 \times 500$ pixels, and the noise-free projection data was measured using a 500 bins detector over $180^{\circ}$ with parallel-beam geometry and simple line-length model. The projection data was measured using 20, 10 and 7 projection views and image reconstruction was implemented using FBP, OS-Convex, ART-TV (Sidky et al 2006) and OS-iMAP methods. The image reconstruction using ART-TV method was implemented through a data enforcement step using the well-known ART algorithm followed by a TV-minimization step in an alternating manner. The parameters of the ART-TV method were optimized to obtain the best image on RMSE scale. For iterative 
(a) FBP
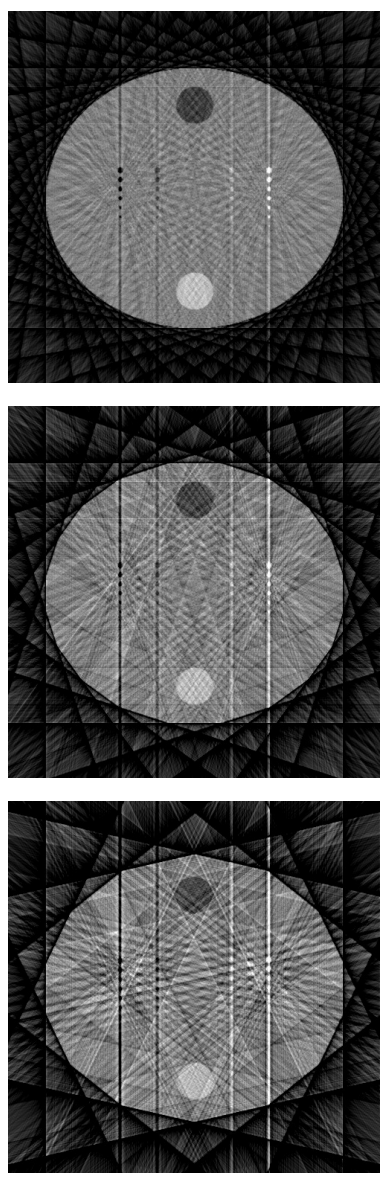

(b) OS-Convex
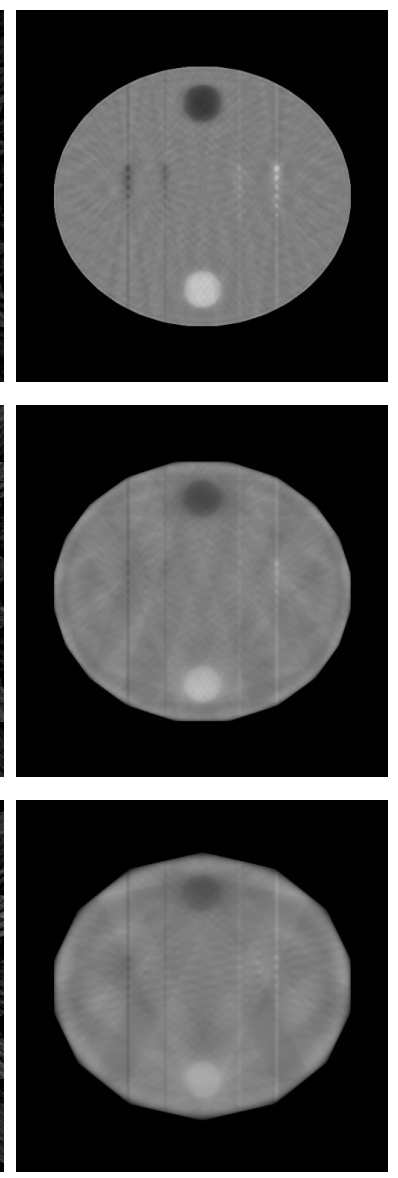

(c) ART-TV
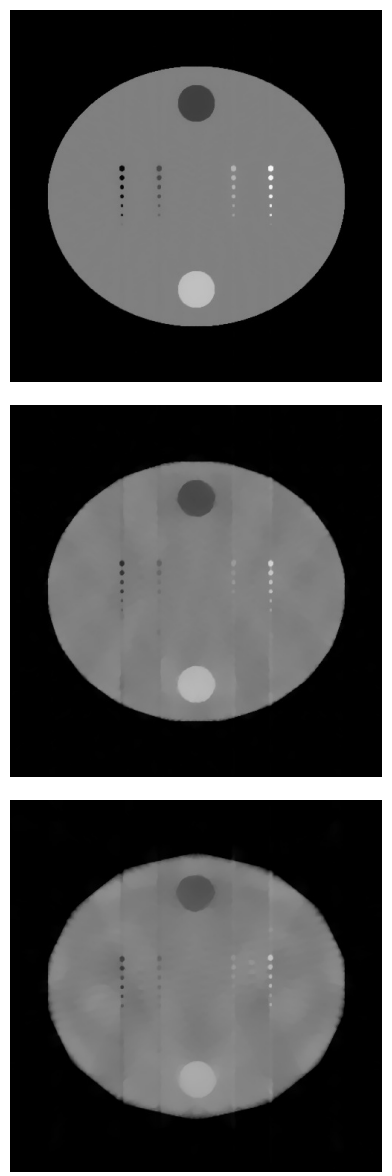

(d) OS- $i$ MAP
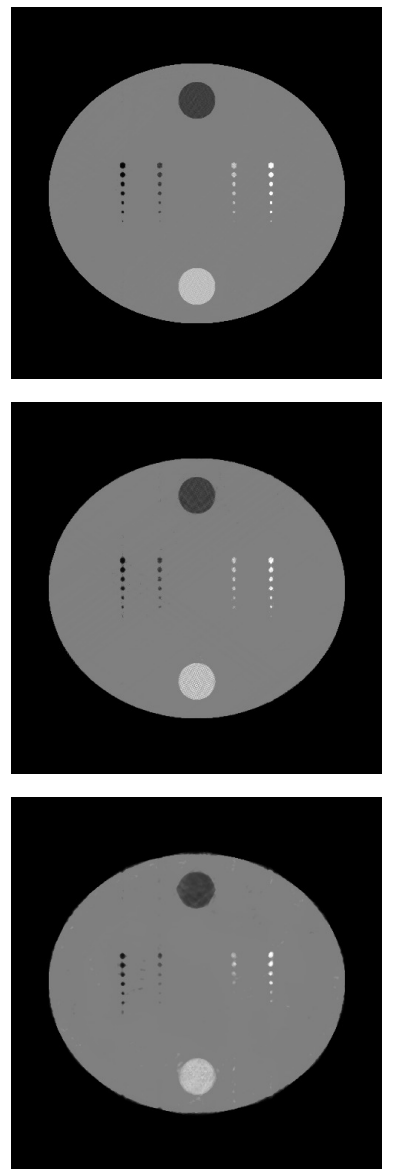

Figure 4. Images reconstructed using (a) standard FBP, (b) OS-Convex, (c) ART-TV and (d) OS-iMAP methods from 20, 10, and 7 projections (from top to bottom).

methods, the iteration number was unified to 100 iterations with initial uniform image computed from projection data. The number of subsets used for the OS-Convex and OS- $i$ MAP methods was set to 5,5 , and 7 subsets for reconstruction from 20, 10, and 7 view angles, respectively.

The intensity prior for the OS- $i$ MAP method was selected to be the intensity values corresponding to air (region outside the object) and the uniform background of the phantom. We assumed that the inserts are unknown structures. This assumption would probably fit with many diagnostic CT applications, where the intensity value of a uniform background is known while lesions are commonly unknown. The reconstruction was implemented with $L=2, \beta=0.008, z=(0.0,1.0) \mathrm{cm}^{-1}$ and $\omega=(0.01,0.06)$. From only 7 projections, the detectability of the inserts are significantly improved by using the proposed method. This can be confirmed by the visibility of the small size inserts shown in figure 4 and the corresponding profile plots in figure 5. Unlike the ART-TV method, it is observed that, the large size inserts at 12 and 6 o'clock positions of the phantom still suffer from weak streak artifacts by using the proposed method. This behavior is expected and the reasons are: (1) we did not include any intensity prior corresponding 

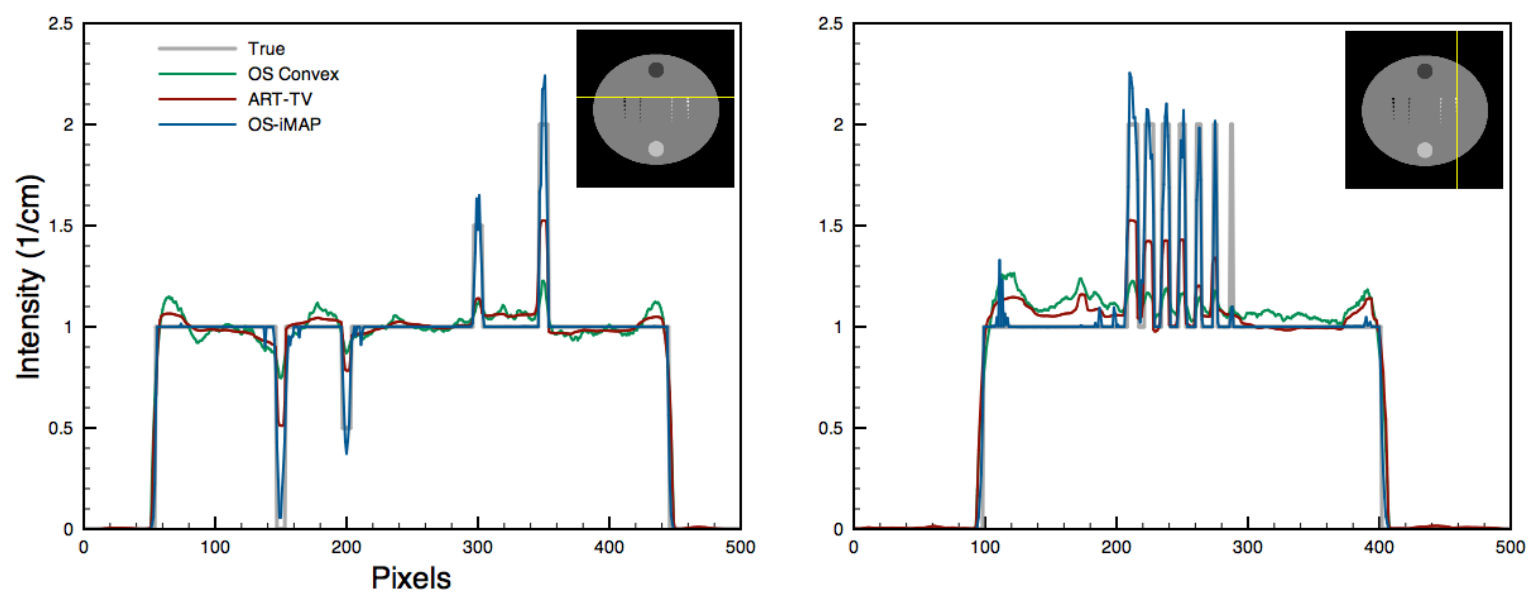

Figure 5. Horizontal and vertical profile plots across the inserts of some results presented in figure 4. Comparison is between images reconstructed from 7 projections.
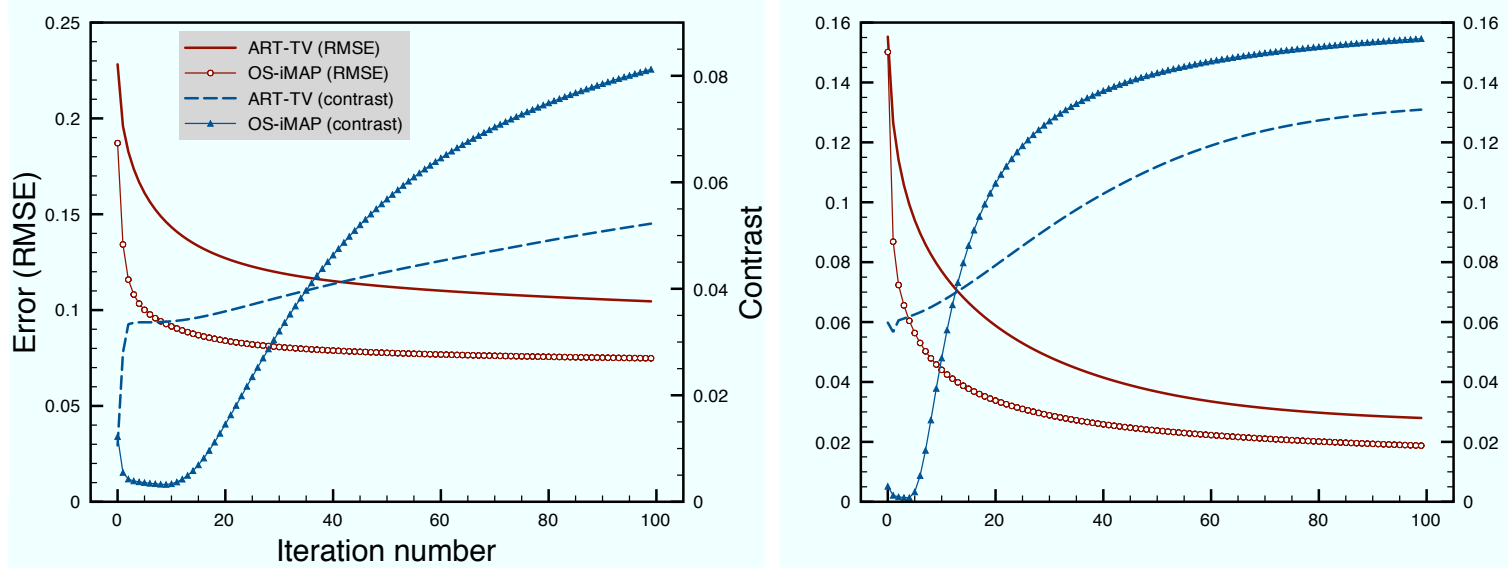

Figure 6. Measured contrast (right vertical axis) of seven inserts, marked by the arrow in figure 3, and RMSE (left vertical axis) for images reconstructed using ART-TV and OS- $i$ MAP methods from 7 (left) and 20 (right) projections.

to the inserts and (2) the objective function does not include any uniformity enforcing term (e.g. smoothing penalty). Including smoothing penalty, however, would probably profit image uniformity in the cost of contrast especially for the small size inserts.

The RMSE and the contrast of the seven inserts, marked by the arrow in figure 3, were computed for the images reconstructed from ART-TV and OS- $i$ MAP methods. Results obtained from 7 and 20 projections are shown in figure 6. From these results, it can be observed that the proposed method achieves a significant improvement in contrast with acceptable RMSE value, especially when the number of projections is highly undersampled. The true contrast of the seven inserts is 0.2 . The same experiment was repeated with added Poisson noise corresponding to $10^{5}$ photon counts and reconstructed images from 25 projections are shown in figure 7.

In another experiment, we investigate the proposed method when the intensity 
(a) FBP

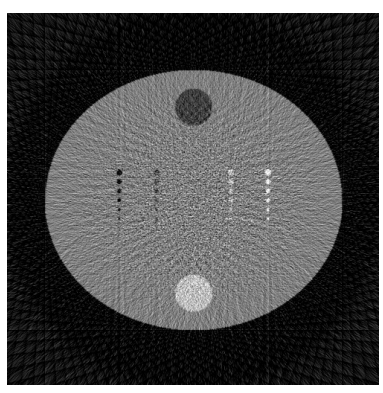

(b) OS-Convex

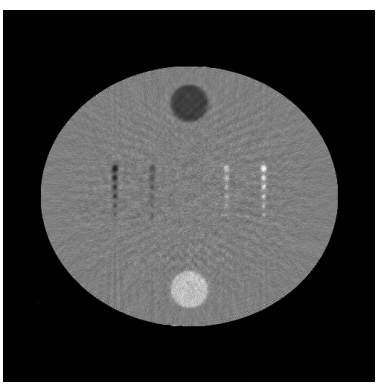

(c) ART-TV

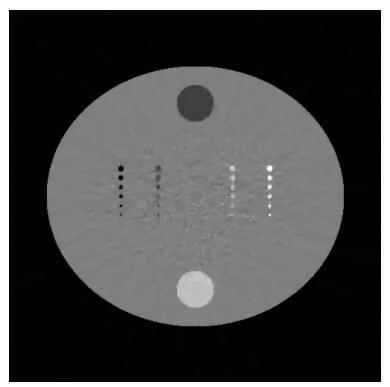

(d) OS- $i$ MAP

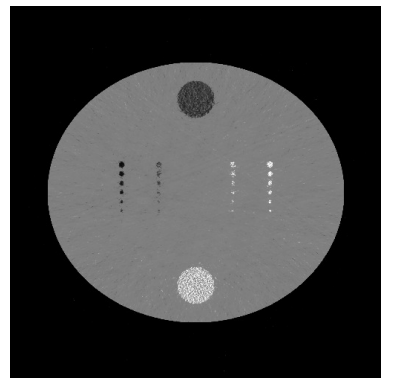

Figure 7. Images reconstructed from 25 projections with added Poisson noise.
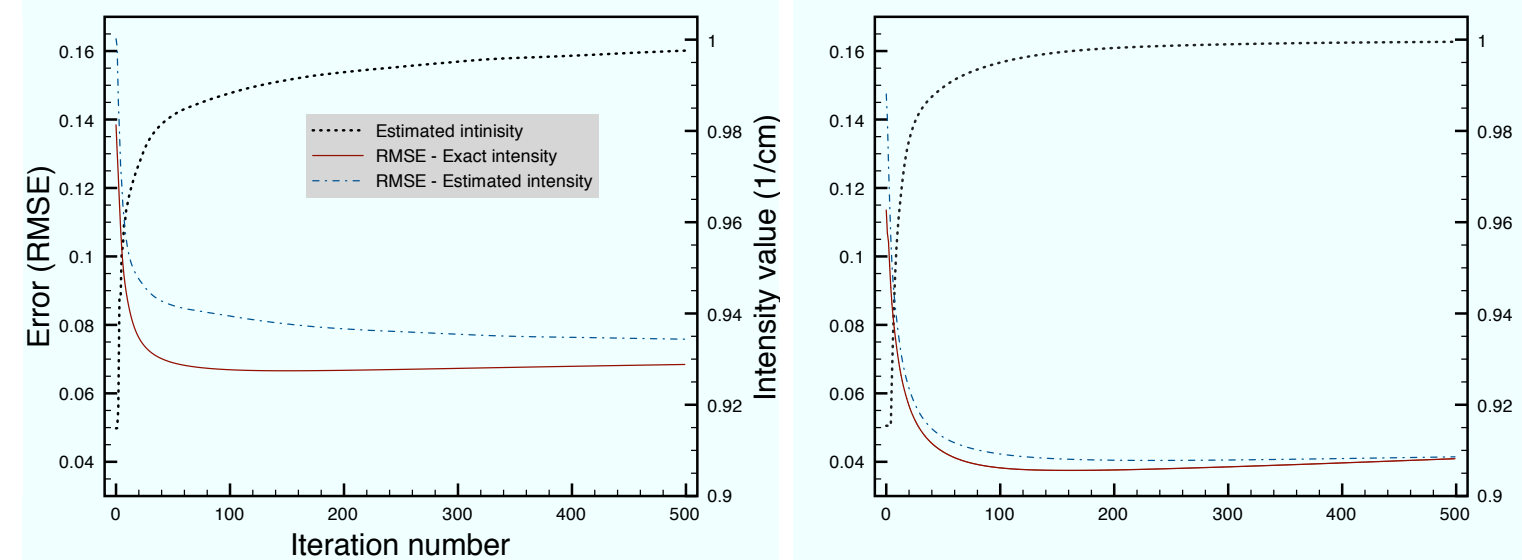

Figure 8. Measured RMSE (left vertical axis) and estimated intensity value (right vertical axis) of the uniform background computed from OS-iMAP reconstruction from 7 (left) and 20 (right) projections.

prior is unavailable and estimated during the reconstruction. The intensity prior were estimated initially from the FBP image and then updated at every iteration using the current image estimate. For simplicity, we limited the estimated intensity prior to only the value corresponding to the uniform background. This value was computed as the median intensity value of the current image estimate. We have used the same experiment settings used in the previous study. The estimated intensity value corresponding to the uniform background and the RMSE of the reconstructed image were calculated for the case of reconstruction from 7 and 20 projections along 500 iterations, and the results are shown in figure 8 . These results indicate that the estimated intensity value is approaching to the accurate one as the iteration proceeds. However, more iterations were required to reach the exact intensity value and, therefore, the same image quality.

The effect of under-relaxation parameter introduced in (22) was evaluated in another experimental study. A noise-free data measured over 20 projection views were used for a reconstruction using different values of the hyper-parameter $\beta$. We have used a static values of $0.1,0.01$ and 0.001 and compare it with the dynamic value used in the above experiment with $\beta=0.008$. Reconstructed images from 100 iterations are shown 
(a) Dynamic, $\beta=0.008$

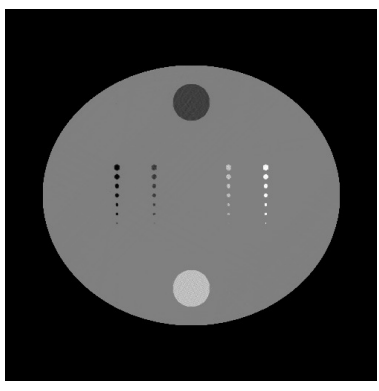

(b) $\beta=0.1$

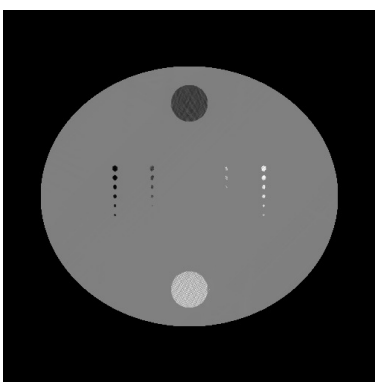

(c) $\beta=0.01$

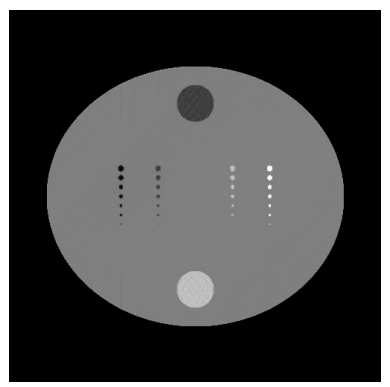

(d) $\beta=0.001$

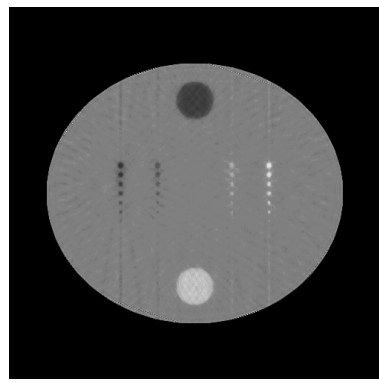

Figure 9. Image reconstructed using OS- $i$ MAP algorithm from 20 projections with (a) dynamic parameter computed using (23) with $\beta=0.008$ and with static values of (b) $\beta=0.1$ (c) $\beta=0.01$ and (d) $\beta=0.001$.
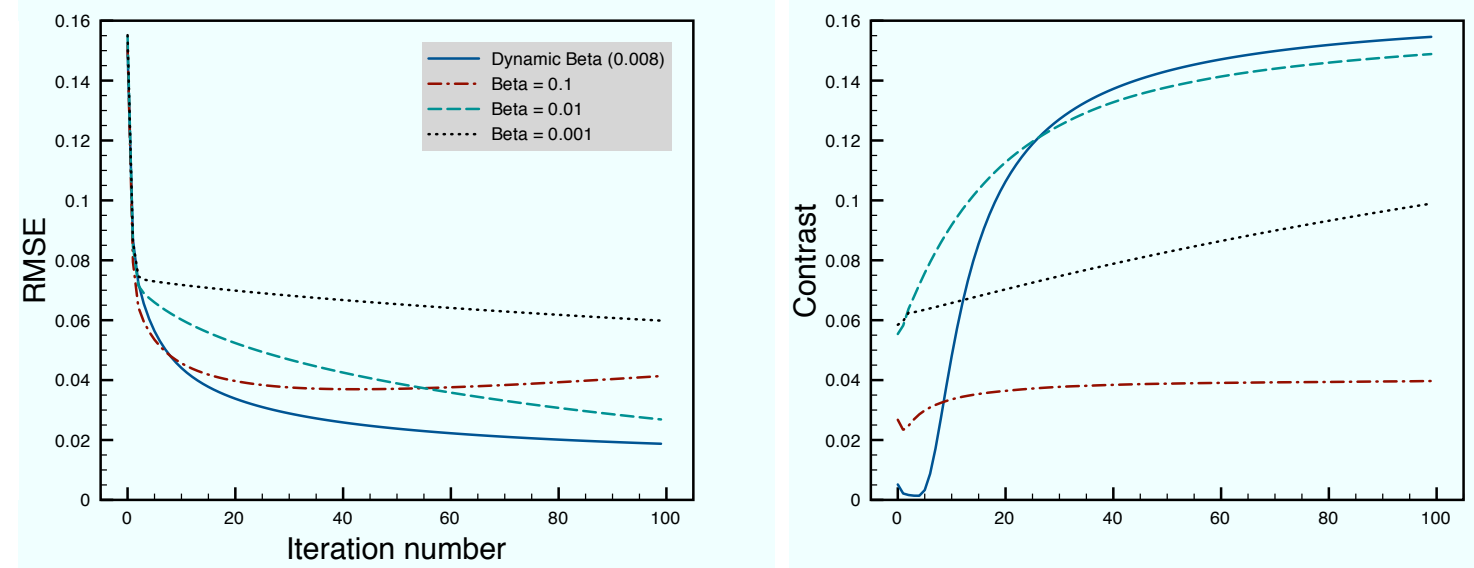

Figure 10. RMSE and contrast computed from images shown in figure 9.

in figure 9 and the measured RMSE and contrast are shown in figure 10. It is observed that, when $\beta$ was set to relatively large value, small size and low-contrast inserts were almost lost. On the other hand, when $\beta$ is relatively small, streak artifacts were not effectively removed. Moreover, even with intermediate optimized value of $\beta=0.01$, the image quality measurements are behind the case where the under-relaxation parameter is used as shown in figure 10.

\subsection{Real data (head CT)}

This experiment was performed using real head CT data obtained from a thirdgeneration scanner. The sampling of the acquired fan-beam data consists of $512($ bins $) \times$ 600 (views) over $360^{\circ}$ which rebinned into a parallel-beam data of 512 (bins) $\times 600$ (views) and then manually down-sampled into 75 projection views over $180^{\circ}$. As the blank scan measurements are unavailable, it is estimated from the raw data measurements and a uniform value of $10^{4}$ was used. This study aims to qualitatively compare the OS$i$ MAP method with the ART-TV method and the conventional FBP. We have limited the number of iterations in both iterative methods to 50 iterations. For the OS- $i$ MAP 
(a) FBP-600

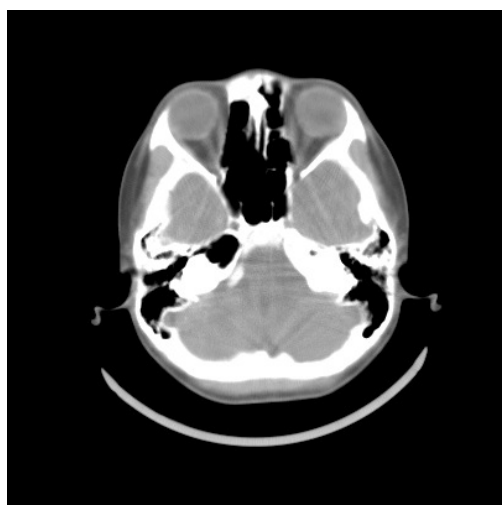

(d) FBP-75

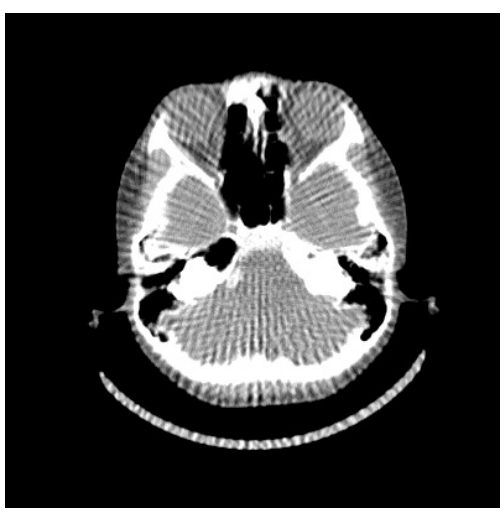

(b) ART-TV-75

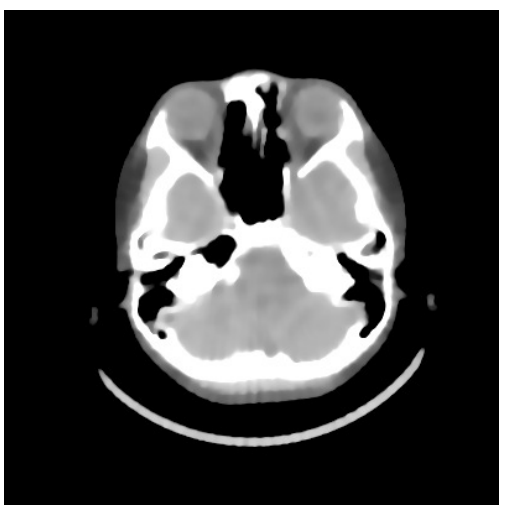

(e) ART-TV-75 (Error)

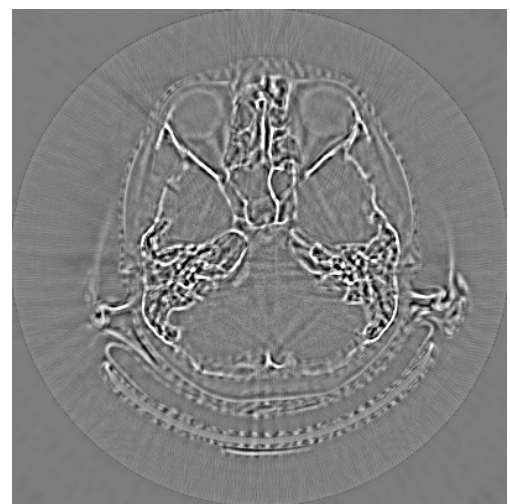

(c) OS- $i$ MAP-75

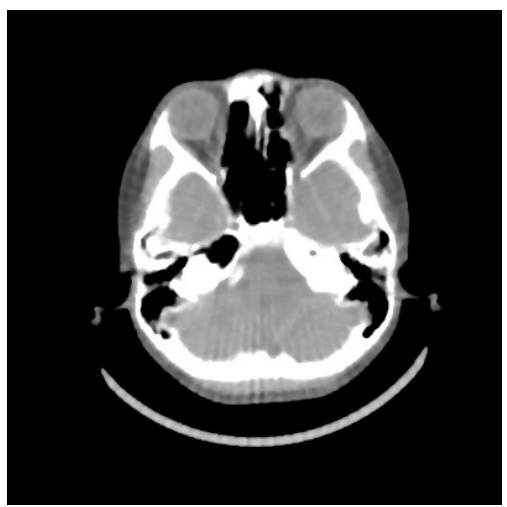

(f) OS- $i$ MAP-75 (Error)

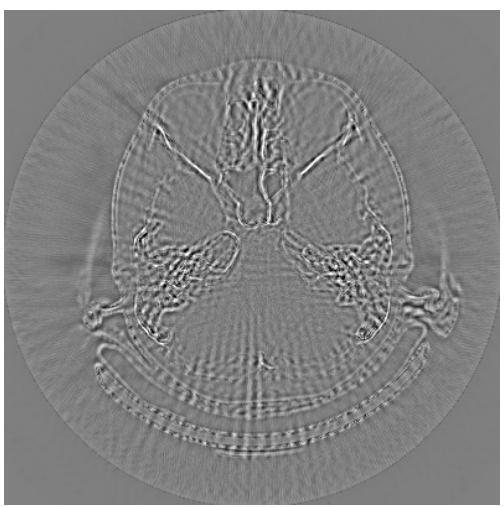

Figure 11. (a) FBP image reconstructed from complete data and images reconstructed from 75 projections using (b) ART-TV, (c) OS-iMAP, and (d) FBP algorithms. Error images in (e) and (f) are computed as the difference between (a), (b) and (a), (c), respectively. Display gray scale is $(0.85,1.15) \mathrm{cm}^{-1}$ except for (e) and $(\mathrm{f})$, it is $(-0.1,0.1) \mathrm{cm}^{-1}$. Displayed images are trimmed to $400 \times 400$ pixels for display magnification.

method, we used $L=3, \beta=0.02, z=(0.0,0.94,1.044) \mathrm{cm}^{-1}$ and $\omega=(0.02,0.02,0.02)$ with 5 subsets. Reconstructed images are shown in figure 11. The proposed method can significantly reduce streak artifacts using a simple and easy-to-compute intensity prior, which were estimated as the histogram peaks of the FBP image shown in figure 11(d). Image reconstructed using ART-TV method yields almost no streak artifacts. However, it is clear from the error image shown in figure 11(e) that a large portion of image contrast in some regions, especially bone, was lost. On the other hand, the image reconstructed from the OS- $i$ MAP method contains weak artifacts, but image contrast is highly preserved as shown by the error image in figure 11(f).

\subsection{Pseudo-real data (chest CT)}

In the pseudo-real data experiment, we used previously reconstructed CT volume to evaluate the proposed method. The original data were scanned using x-ray power of $120 \mathrm{KeV}$ and reconstructed in a volume grid of $512 \times 512 \times 77$ with $0.63 \mathrm{~mm}$ pixel size 
(a) FBP-original
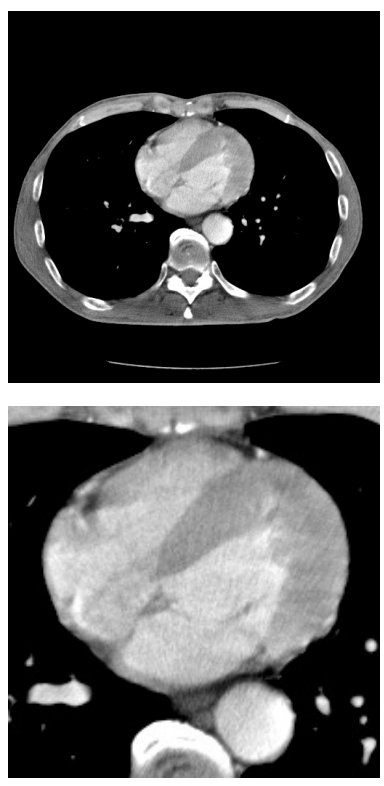

(b) FBP-100
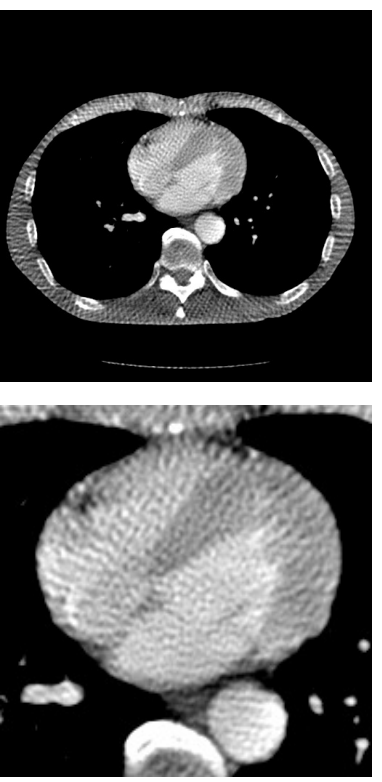

(c) ART-TV-100
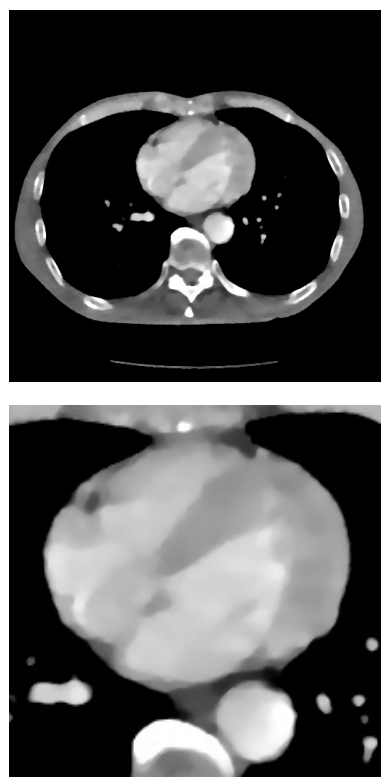

(d) OS- $i$ MAP-100
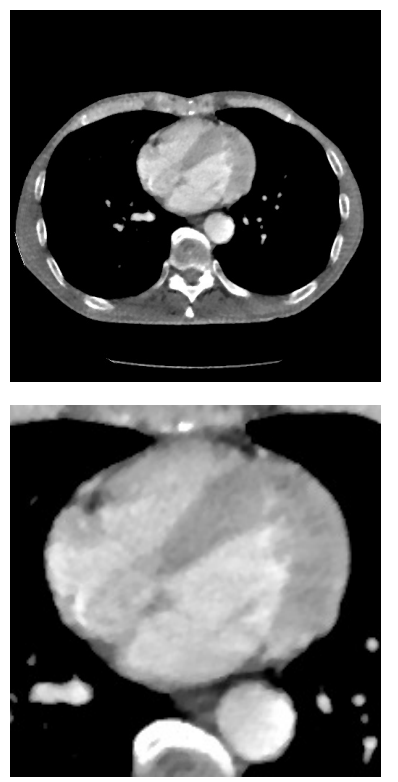

Figure 12. Image reconstruction of the chest $\mathrm{CT}$ data. (a) Original slice image and reconstructions from 100 projections using (b) FBP, (c) ART-TV and (d) OS-iMAP methods. A magnification of the cardiac region is shown in the bottom row. Display gray scale is $(0.85,1.3) \mathrm{cm}^{-1}$.

and slice size of $1.0 \mathrm{~mm}$. A single slice was re-projected into 100 projection views over $180^{\circ}$ and reconstruction was implemented using FBP, ART-TV and OS- $i$ MAP methods. The histogram of the FBP image was used to estimate the intensity prior to be used in the OS- $i$ MAP method. We selected four histogram peaks that correspond to the average intensity values of air, lung, soft-tissue and blood. The experiment settings for the OS- $i$ MAP method were $L=4, \beta=0.02, z=(0.0,0.104,1.015,1.05) \mathrm{cm}^{-1}$ and $\omega=(0.04,0.1,0.03,0.02)$ with 5 subsets. A uniform value of $10^{5}$ was used as the blank scan measurement. The reconstruction was implemented using 50 iterations and the results are shown in figure 12. It is observed that the FBP image suffers from sever streak artifacts, which are significantly reduced in the ART-TV and OS- $i$ MAP images. However, the well-known carton-like effect of the TV-minimization is avoided in the image reconstructed using the proposed method. Although, the OS- $i$ MAP image still suffer from weak artifacts especially in peripheral regions, we expect that these artifacts can be effectively removed with further iterations.

\subsection{Real data (high-resolution CT)}

In this experiment, we have used a three-dimensional high-resolution CT data obtained from SPring-8 BL20XU beam line. SPring-8 is a third-generation synchrotron radiation source located in Hyogo, Japan (http://www.spring8.or.jp). The imaged object is a micro sample of aluminum alloy $(A l-4.4 \% C u)$. In this application, it is desirable to obtain high-quality images from a small number of projection views as the acquired 


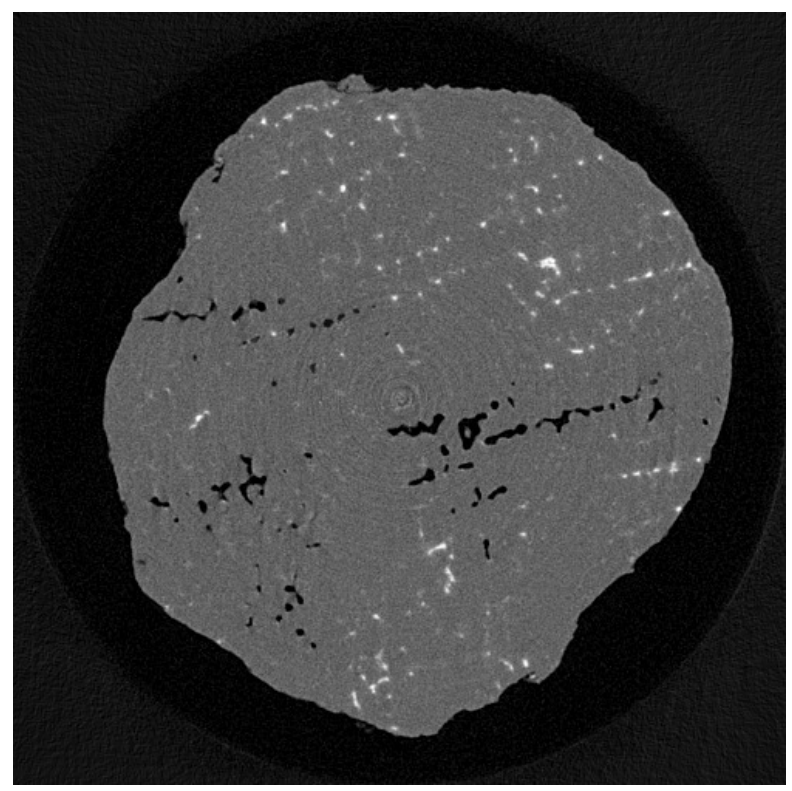

Figure 13. Central slice of the micro metal alloy reconstructed from 1500 projection views using FBP algorithm.

projection data are huge and 3D reconstruction is computationally expensive. The sample of $1 \mathrm{~mm}$ diameter was fully scanned with x-ray energy of $35 \mathrm{KeV}$. The projection data were obtained using a charge-coupled device (CCD) camera of $4000 \times 2624$ bins with $2 \times 2$ binning mode an $5.9 \times 5.9 \mu \mathrm{m}$ pixel size. The original parallel-beam projection data used in this experiment were acquired over uniformly spaced 1500 view angles over $180^{\circ}$ with exposure time of $300 \mathrm{~ms} /$ view. Two blank scans were obtained through preand post- data measurements. The detector array was resampled into $500 \times 328$ bins to reduce the computation cost for image reconstruction. The target of this imaging application is to investigate the fine structures of cracks and air holes inside the metal sample, as well as, the distribution of different components of the alloy. A central slice, reconstructed from 1500 projection views using FBP algorithm, is shown in figure 13.

The projection data was manually down-sampled to 150, 100 and 50 projection views and reconstruction was implemented using FBP, ART-TV and OS- $i$ MAP methods. We have used 8, 12 and 16 iterations for the OS- $i$ MAP algorithm to reconstruct images from 150, 100 and 50 projections, respectively. Almost the same number of iterations was used to obtain the ART-TV image with highest image quality. We have used average intensity values of the homogenous background (Aluminum) and air as the known intensity values. The parameters for the OS- $i$ MAP method were set to $L=2$, $\beta=0.06, z=(0.0,2.7) \mathrm{cm}^{-1}$ and $\omega=(0.01,0.06)$. The reconstruction from 150 and 100 projection views are shown in figure 14 and 3D reconstructions of the whole object from 50 projections are shown in figure 15. These results indicate that the image quality is significantly improved by employ the proposed method. Air holes and high contrast metals are clearly observed even by using highly under-sampled projection data. 
(a) FBP-150

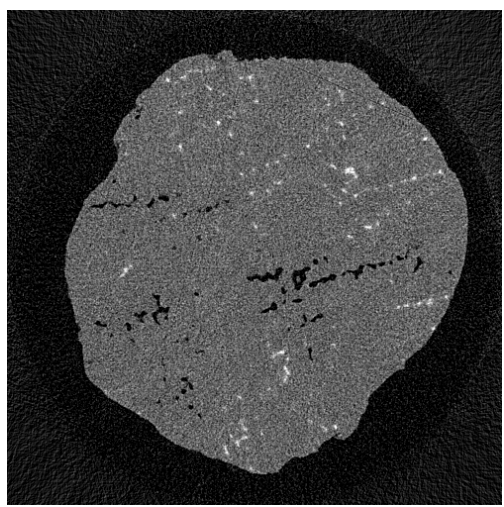

(d) FBP-100

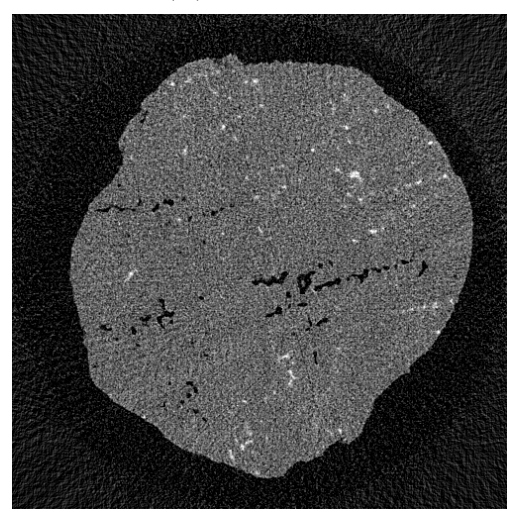

(b) ART-TV-150

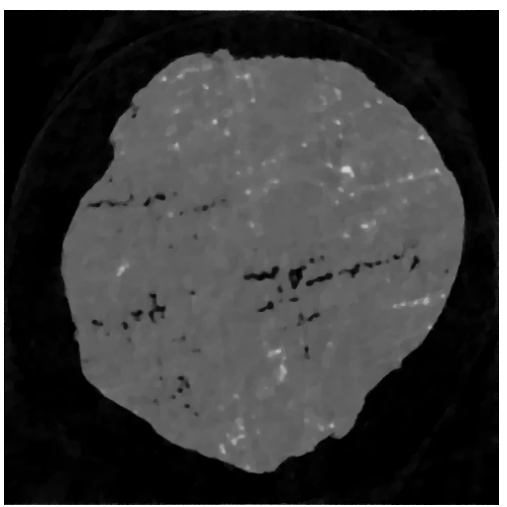

(e) ART-TV-100

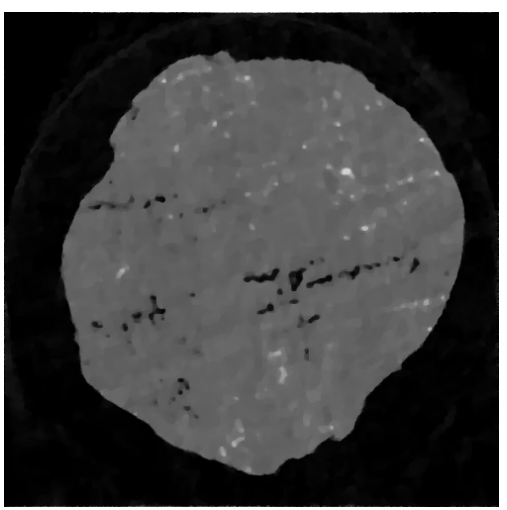

(c) OS- $i$ MAP- -150

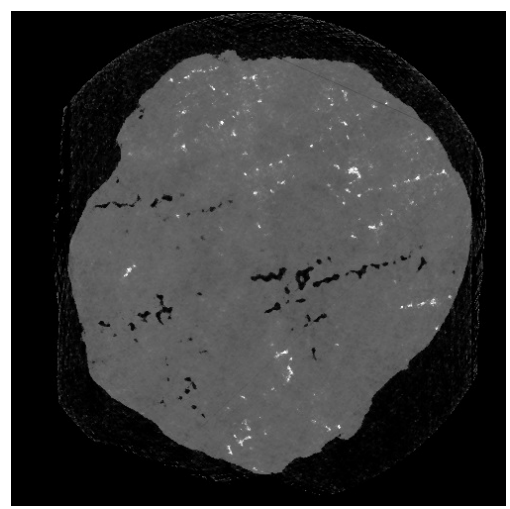

(f) OS- $i$ MAP-100

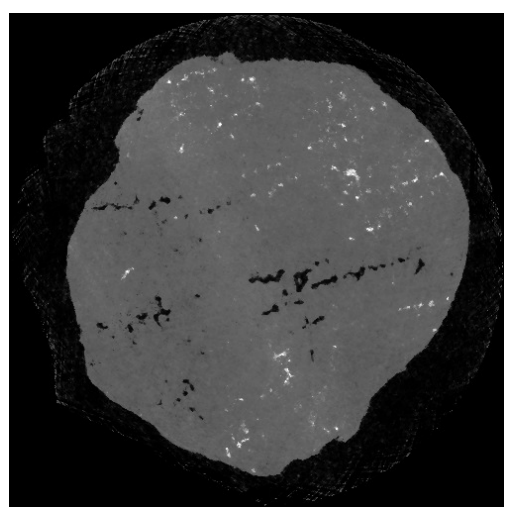

Figure 14. Reconstructed images of the micro metal alloy using FBP (left), ART-TV (middle) and OS-iMAP (right) from 150 (top) and 100 (bottom) projection views.

\section{Discussion and Conclusion}

In this paper, we present a framework for SR from a small number of projection views in x-ray CT. The main contribution is the use of intensity prior as a penalty term in the objective function for image reconstruction. The required intensity prior is represented by a small number of intensity values that associated to the homogenous regions expected in the scanned object. The proposed $i \mathrm{MAP}$ algorithm can be easily implemented through a single step of conventional reconstruction followed by a multithresholding step in an alternating manner. Moreover, several possible reconstruction algorithms can be derived using the same approach presented here.

The efficiency of the $i$ MAP algorithm is compared to recently developed image reconstruction method based on TV-minimization, which is used to solve the problem of image reconstruction from a small number of projections. Both approaches, the $i \mathrm{MAP}$ and the ART-TV methods, outperform conventional analytical methods such as FBP in terms of streak artifacts reduction. However, small size and/or low contrast structures tend to be lost when the TV-minimization is employed, especially when the number of projection views is highly under-sampled, e.g. less than 10 projections. It is also known that, in some cases, the TV-minimization may lead to the loss of considerable fraction 
(a) FBP-50
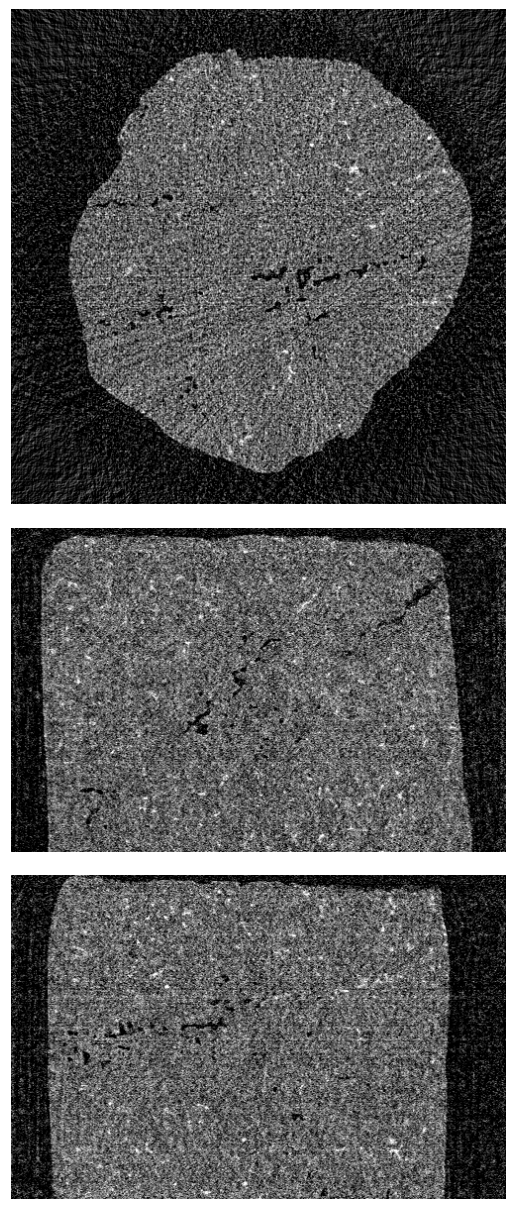

(b) ART-TV-50
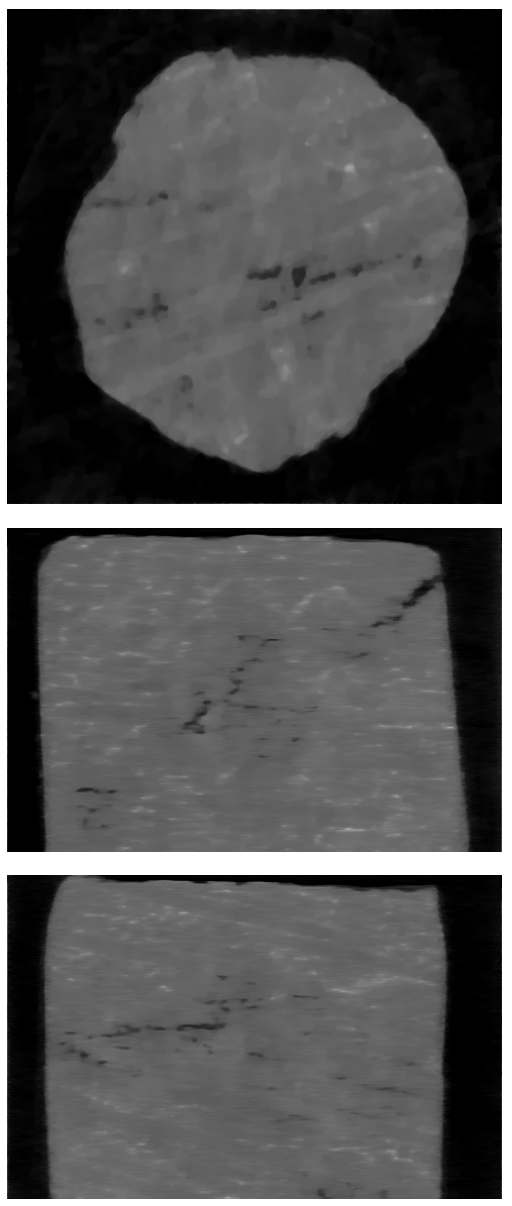

(c) OS- $i$ MAP-50
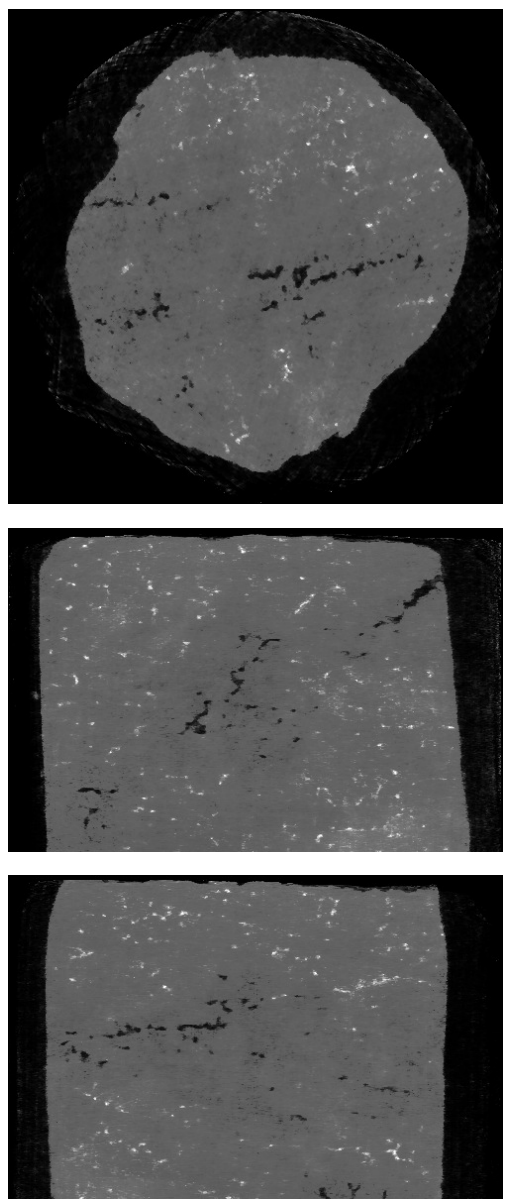

Figure 15. 3D Reconstruction of micro metal alloy using FBP (left), ART-TV (middle) and OS-iMAP (right) from 50 projection views. Central transverse, coronal and sagittal slices are shown from up to bottom, respectively.

of the object contrast. Experimental results indicate that $i \mathrm{MAP}$ method effectively preserves small size and/or low contrast image details while achieving high image contrast. Even with real data, where the measured intensity values within the same region is not strictly uniform; the proposed approach outperforms TV-minimization in terms of image quality. In addition, the resulting image does not include the carton-like image effect known for the TV-minimization methods.

An important factor in the proposed approach is how to control the hyper-parameter $\beta$. During the preliminary experimental studies, we have found that if $\beta$ is used as a fixed value during iterative reconstruction, the quality of reconstructed image becomes sensitive to the value of $\beta$. For example, if $\beta$ is selected as a relatively large value, some fine image details would probably lost. On the other hand, if $\beta$ is selected as a relatively small value, the effect of multi-thresholding is weakened and the proposed method behaves similar to conventional methods. The use of under-relaxation approach, such that we start with a relatively large value that gradually decrease with iteration, provides a useful and effective choice. A strong thresholding is required in early iterations to 
remove streak artifacts and weak thresholding is required later to enforce the data fidelity term to retrieve any possible missing structures.

The proposed method has a large possibility to be used in several low-dose x-ray CT imaging applications. Moreover, the proposed framework is general in such a way, it has a potential to be used in the other data limitation problems. It is interesting to investigate these problems in future work. However, we expect that its validity and power strongly depend on the type of image artifacts to be eliminated using intensity prior.

\section{Acknowledgments}

First author acknowledges support from Japan Society for the Promotion of Science (JSPS) postdoctoral fellowship (ID No. P10052). The authors are very grateful to Dr. Hiroyuki Toda (Toyohashi University of Technology, Japan) for providing the highresolution x-ray CT data, and to the referees for their useful comments.

\section{Appendix A. Derivation of equation (21)}

For simplicity, let us consider that $L=1$. Following equations (19) and (20), the minimization of the $\tilde{f}_{\beta}\left(\mu ; \mu^{k}\right)$ in equation (18) is given as:

$$
\begin{aligned}
q_{j} & = \begin{cases}p_{j}+1 /\left(2 c_{j, 1}\right) & \left(p_{j}<z_{1}-1 /\left(2 c_{j, 1}\right)\right) \\
z_{1} & \left(z_{1}-1 /\left(2 c_{j, 1}\right) \leq p_{j} \leq z_{1}+1 /\left(2 c_{j, 1}\right)\right) \\
p_{j}-1 /\left(2 c_{j, 1}\right) & \left(p_{j}>z_{1}+1 /\left(2 c_{j, 1}\right)\right)\end{cases} \\
& = \begin{cases}p_{j}+1 /\left(2 c_{j, 1}\right) & \left(p_{j}<z_{j, 1}^{-}\right) \\
z_{1} & \left(z_{j, 1}^{-} \leq p_{j} \leq z_{j, 1}^{+}\right) \\
p_{j}-1 /\left(2 c_{j, 1}\right) & \left(p_{j}>z_{j, 1}^{+}\right)\end{cases}
\end{aligned}
$$

with $z_{j, 1}^{+}=z_{1}+1 /\left(2 c_{j, 1}\right), z_{j, 1}^{-}=z_{1}-1 /\left(2 c_{j, 1}\right)$. Similarly, the general multi-thresholding function for $l=(1, \ldots, L)$ is given as:

$$
q_{j}= \begin{cases}p_{j}+1 /\left(2 c_{j, 1}\right) & \left(p_{j}<z_{j, 1}^{-}\right) \\ z_{1} & \left(z_{j, 1}^{-} \leq p_{j} \leq \min \left(z_{j, 1}^{+}, s_{1}\right)\right) \\ p_{j}-1 /\left(2 c_{j, 1}\right) & \left(z_{j, 1}^{+}<p_{j} \leq s_{1}\right) \\ \vdots & \vdots \\ p_{j}+1 /\left(2 c_{j, l}\right) & \left(s_{l-1}<p_{j}<z_{j, l}^{-}\right) \\ z_{l} & \left(\max \left(z_{j, l}^{-}, s_{l-1}\right) \leq p_{j} \leq \min \left(z_{j, l}^{+}, s_{l}\right)\right) \quad,(l=2, \ldots, L-1) \\ p_{j}-1 /\left(2 c_{j, l}\right) & \left(z_{j, l}^{+}<p_{j} \leq s_{l}\right) \\ \vdots & \vdots \\ p_{j}+1 /\left(2 c_{j, L}\right) & \left(s_{L-1}<p_{j}<z_{j, L}^{-}\right) \\ z_{L} & \left(\max \left(z_{j, L}^{-}, s_{L-1}\right) \leq p_{j} \leq z_{j, L}^{+}\right) \\ p_{j}-1 /\left(2 c_{j, L}\right) & \left(z_{j, L}^{+}<p_{j}\right)\end{cases}
$$


where $s_{l}, l=(1, \ldots, L-1)$ is the intersection point between the successive $\ell_{1}$ norms around intensity values $z_{l}$ and $z_{l+1}$ and is given by:

$$
s_{l}=\left(\omega_{l} z_{l}+w_{l+1} z_{l+1}\right) /\left(\omega_{l}+\omega_{l+1}\right) .
$$

Therefore, the multi-thresholding function in equation (A.3) can be written in the following simple form:

$q_{j}= \begin{cases}p_{j}+1 /\left(2 c_{j, l}\right) & \left(s_{l-1}<p_{j}<z_{j, l}^{-}\right) \\ z_{l} & \left(\max \left(z_{j, l}^{-}, s_{l-1}\right) \leq p_{j} \leq \min \left(z_{j, l}^{+}, s_{l}\right)\right) \quad,(l=1, \ldots, L), \\ p_{j}-1 /\left(2 c_{j, l}\right) & \left(z_{j, l}^{+}<p_{j} \leq s_{l}\right)\end{cases}$

with $s_{0}=-\infty$ and $s_{L}=\infty$.

\section{References}

Beekman F J and Kamphuis C 2001 Ordered subsets reconstruction for x-ray CT Phys. Med. Biol. 46 $1835-44$

Bian J, Siewerdsen J H, Han X, Sidky E Y, Prince J L, Pelizzari C A and Pan X 2010 Evaluation of sparse-view reconstruction from flat-panel-detector cone-beam CT Phys. Med. Biol. 55 6575-99

Blumensath T and Davies M E 2010 Normalized iterative hard thresholding: guaranteed stability and performance IEEE J. Sel. Topics Signal Process 4 298-309

Brenner D J and Hall E J 2007 Computed Tomography - An increasing source of radiation exposure N. Engl. J. Med. 357 2277-84

Brooks R A, Glover G, Talbert A J, Eisner R L and DiBianca F A 1979 Aliasing: a source of streak in computed tomograms J. Comput. Assist. Tomogr. 3 511-8

Candès E J, Romberg J and Tao T 2006 Robust uncertainty principles: exact signal reconstruction from highly incomplete frequency information IEEE Trans. Inf. Theory $\mathbf{5 2}$ 489-509

Chen G-H, Tang J and Leng S 2008 Prior image constrained compressed sensing (PICCS): A method to accurately reconstruct dynamic CT images from highly undersampled projection data sets $M e d$. Phys. 35 660-3

Daubechies I, Defrise M and De Mol C 2004 An Iterative thresholding algorithm for linear inverse problems with a sparsity constraint Comm. Pure Appl. Math. 57 1413-57

Donoho D L 2006 Compressed sensing IEEE Trans. Inf. Theory 52 1289-306

Erdǒgan H, Fessler J A 1999 Monotonic algorithms for transmission tomography IEEE Trans. Med. Imag. 18 801-14

Fahimian B P, Mao Y, Cloetens P and Miao J 2010 Low-dose x-ray phase-contrast and absorption CT using equally sloped tomography Phys. Med. Biol. 55 5383-400

Fessler J A and Hero A O 1995 Penalized maximum-likelihood image reconstruction using spacealternating generalized EM algorithms IEEE Trans. Image Process. 4 1417-29

Galigekere R R, Wiesent K and Holdsworth D W 1999 Techniques to alleviate the effects of view aliasing artifacts in computed tomography Med. Phys. 26 896-904

Hansis E, Schäfer D, Dössel O and Grass M 2008 Evaluation of iterative sparse object reconstruction from few projections for 3-D rotational coronary angiography IEEE Trans. Med. Imag. 27 1548-55

Herman G T and Davidi R 2008 Image reconstruction from a small number of projections Inverse Problems 24045011 (17pp)

Herman G T and Kuba A 1999 Discrete Tomography: Foundations, Algorithms and Applications (Boston, MA: Birkhäuser)

Herman G T and Kuba A 2007 Advances in Discrete Tomography and Its Applications (Boston, MA: Birkhäuser) 
Huesman R H 1977 The effects of a finite number of projection angles and finite lateral sampling of projections on the propagation of statistical errors in transverse section reconstruction Phys. Med. Biol. 22 511-21

Joseph P M and Schulz R A 1980 View sampling requirements in fan beam computed tomography Med. Phys. 7 692-702

Kole J S 2005 Statistical image reconstruction for transmission tomography using relaxed ordered subset algorithms Phys. Med. Biol. 50 1533-45

Kolehmainen V, Siltanen S, Järvenpää S, Kaipio J P, Koistinen P, Lassas M, Pirttilä J and Somersalo E 2003 Statistical inversion for medical x-ray tomography with few radiographs: II. Application to dental radiology Phys. Med. Biol. 48 1465-90

Lange K, Bahn M and Little R 1987 A theoretical study of some maximum likelihood algorithms for emission and transmission tomography IEEE Trans. Med. Imag. 6 106-14

Lange K and Carson R 1984 EM reconstruction algorithms for emission and transmission tomography J. Comput. Assist. Tomogr 8 306-16

Lange K and Fessler J A 1995 Globally convergent algorithms for maximum a posteriori transmission tomography IEEE Trans. Image Process 4 1430-8

Leng S, Tang J, Zambelli J, Nett B, Tolakanahalli R and Chen G-H 2008 High temporal resolution and streak-free four-dimensional cone-beam computed tomography Phys. Med. Biol. 53 5653-73

Li M, Kudo H, Hu J and Johnson R H 2004 Improved iterative algorithm for sparse object reconstruction and its performance evaluation with micro-ct data IEEE Trans. Nucl. Sci. 51 659-66

Li M, Yang H and Kudo H 2002 An accurate iterative reconstruction algorithm for sparse objects: application to 3D blood-vessel reconstruction from a limited number of projections Phys. Med. Biol. 47 2599-609

Loris I 2009 On the performance of algorithms for the minimization of $\ell_{1}$-penalized functionals Inverse Problems 25035008 (16pp)

Mameuda Y and Kudo H 2007 New anatomical-prior-based image reconstruction method for PET/SPECT Conference Record of 2007 IEEE Nuclear Science Symposium and Medical Imaging Conference (Honolulu, USA) Paper No M23-2

Nassi M, Brody W R, Medoff B P and Macovski A 1982 Iterative reconstruction-reprojection: an algorithm for limited data cardiac-computed tomography IEEE Trans. Biomed. Eng. 29 333-41

Natterer F 1986 The mathematics of computerized tomography (New York: Wiley)

Ohnesorge B M, Flohr T G, Becker C R, Knez A and Reiser M F 2006 Multi-slice and dual-source CT in cardiac imaging: principles - protocols - indications - outlook (Berlin: Springer)

Payot E, Prêteux F J, Trousset Y and Guillemaud R. 1997 Generalized support constraint for threedimensional reconstruction from incomplete Fourier spectra J. Electron. Imaging 6 426-38

Persson M, Bone D and Elmqvist H 2001 Total variation norm for three-dimensional iterative reconstruction in limited view angle tomography Phys. Med. Biol. 46 853-66

Rangayyan R, Dhawan A P and Gordon R 1985 Algorithms for limited-view computed tomography: an annotated bibliography and a challenge Appl. Opt. 24 4000-12

Rashed E A and Kudo H 2011 Row-action image reconstruction algorithm using $\ell_{p}$-norm distance to a reference image Conference Record of 2011 IEEE Nuclear Science Symposium and Medical Imaging Conference (Valencia, Spain) Paper No MIC19-3

Rudin L I, Osher S and Fatemi E 1992 Nonlinear total variation based noise removal algorithms Phys. D $60259-68$

Sauer K, James S Jr and Klifa C 1994 Bayesian estimation of 3-D objects from few radiographs IEEE Trans. Nucl. Sci. 41 1780-90

Sidky E Y, Kao C-M and Pan X 2006 Accurate image reconstruction from few-views and limited-angle data in divergent-beam CT J. X-Ray Sci. Technol. 14 119-39

Sidky E Y and Pan X 2008 Image reconstruction in circular cone-beam computed tomography by constrained, total-variation minimization Phys. Med. Biol. 53 4777-807

Siltanen S, Kolehmainen V, Järvenpää S, Kaipio J P, Koistinen P, Lassas M, Pirttilä J and Somersalo 
E 2003 Statistical inversion for medical X-ray tomography with few radiographs: I. General theory Phys. Med. Biol. 48 1437-63

Song J, Liu Q H, Johnson G A and Badea C T 2007 Sparseness prior based iterative image reconstruction for retrospectively gated cardiac micro-ct Med. Phys. 34 4476-83

Tang J, Nett B E and Chen G-H 2009 Performance comparison between total variation (TV)-based compressed sensing and statistical iterative reconstruction algorithms Phys. Med. Biol. 54 5781-804

Yu L, Liu X, Leng S, Kofler J M, Ramirez-Giraldo J C, Qu M, Christner J, Fletcher J G and McCollough C H 2009 Radiation dose reduction in computed tomography: techniques and future perspective Imaging in Medicine 1 65-84 